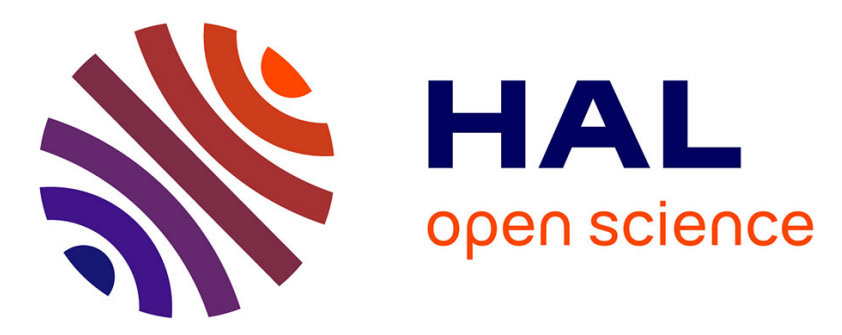

\title{
Analysis of steady-state response regimes of a helicopter ground resonance model including a nonlinear energy sink attachment
}

\author{
Baptiste Bergeot, S Bellizzi, B Cochelin
}

\section{- To cite this version:}

Baptiste Bergeot, S Bellizzi, B Cochelin. Analysis of steady-state response regimes of a helicopter ground resonance model including a nonlinear energy sink attachment. International Journal of NonLinear Mechanics, 2016, 78, pp.72-89. 10.1016/j.ijnonlinmec.2015.10.006 . hal-01091763v5

\section{HAL Id: hal-01091763 \\ https://hal.science/hal-01091763v5}

Submitted on 30 Dec 2017

HAL is a multi-disciplinary open access archive for the deposit and dissemination of scientific research documents, whether they are published or not. The documents may come from teaching and research institutions in France or abroad, or from public or private research centers.
L'archive ouverte pluridisciplinaire HAL, est destinée au dépôt et à la diffusion de documents scientifiques de niveau recherche, publiés ou non, émanant des établissements d'enseignement et de recherche français ou étrangers, des laboratoires publics ou privés. 


\title{
Analysis of steady-state response regimes of a helicopter ground resonance model including a nonlinear energy sink attachment
}

\author{
B. Bergeot ${ }^{a, b, *}$, S. Bellizzi ${ }^{b}$, B. Cochelin ${ }^{b}$ \\ ${ }^{a}$ INSA Centre Val de Loire, Université François Rabelais de Tours, LMR EA 2640, Campus de Blois, 3 Rue de la Chocolaterie, CS 23410, \\ 41034 Blois Cedex, France \\ ${ }^{b}$ LMA, CNRS UPR7051, Aix-Marseille Univ, Centrale Marseille, F-13402 Marseille Cedex 20, France \\ * Corresponding author: baptiste.bergeot@insa-cvl.fr
}

\begin{abstract}
Helicopter Ground Resonance is a dynamic instability involving the coupling of the blades motion in the rotational plane (i.e. the lag motion) and the motion of the fuselage. This paper presents a study of the capacity of a Nonlinear Energy Sink to control a Helicopter Ground Resonance. A model of helicopter with a minimum number of degrees of freedom that can reproduce Helicopter Ground Resonance instability is obtained using successively Coleman transformation and binormal transformation. A theoretical/numerical analysis of the steady-state responses of this model when a Nonlinear Energy Sink is attached on the fuselage in an ungrounded configuration is performed. The analytic approach is based on complexification-averaging method together with geometric singular perturbation theory. Four steady-state responses are highlighted and explained analytically: complete suppression, partial suppression through strongly modulated response, partial suppression through periodic response and no suppression of the Helicopter Ground Resonance. A systematic method based on simple analytical criterions is proposed to predict the steady-state response regimes. The method is finally validated numerically.
\end{abstract}

Keywords: Helicopter ground resonance, Passive control, Non linear Energy Sink, Relaxation oscillations, Strongly modulated response.

\section{Introduction}

Ground Resonance (GR) is a potential destructive mechanical instability that can occur when a helicopter is on the ground and the rotor rotates. The phenomenon of GR involves a coupling between the fuselage motion on its landing gear and the blades motion in the rotational plane (i.e. the lag motion). It can be investigated without taking into account the aerodynamical effects. The standard reference of the GR analysis is the paper by Coleman and Feingold [1] where it is established that GR is due to a frequency coalescence between a lag mode and the fuselage mode. The range of rotors speeds $\Omega$ for which this frequency coalescence occurs is predicted analytically. More references can be found in $[2,3,4]$ and a recent analysis of helicopter GR with asymmetric blades can be found in [5]. Traditionally, GR instability is prevented by two passive methods: increasing the damping [6] and modify the stiffness of the rotor blade lag mode or the fuselage mode. Active control of GR has been also studied in [4].

The Targeted Energy Transfer (TET) concept consists in controlling resonance by using an additional essentially nonlinear attachment also named Nonlinear Energy Sink (NES) to an existing primary linear system. TET has been extensively studied numerically, theoretically and expermentally, the results prove that the NES is very efficient for vibration mitigation [7] and noise reduction [8]. Impulsive loading was theoretically analyzed for example in [9] where TET is investigated in terms of resonance capture. In [10], harmonic forcing was considered where response regimes are characterized in terms of periodic and strongly modulated responses using an asymptotic analysis (multi scale approach) of the averaged flow obtained using the complexification-averaging method [11]. In [12] a NES is used to reduce chatter vibration in turning process. An application of NES as a nonlinear vibration absorber in rotor dynamics can be found in [13] where the efficiency of a collection of NES is analyzed for vibration mitigation of a rotating system under mass eccentricity force.

NESs are also used to control dynamic instabilities. The possible suppression of the limit cycle oscillations of a Van der Pol oscillator utilizing a NES is demonstrated numerically in [14]. In [15] (resp. [16]), the self-excitation response regimes of a Van der Pol (resp. Van der Pol-Duffing) oscillator with a NES are investigated. An asymptotic analysis of the system related to slow/super-slow decomposition of the averaged flow reveals periodic responses, global bifurcations of different types and basins of attraction of various self-excitation regimes. A series of papers [17, 18, 19] demonstrated that a NES coupled to a rigid wing in subsonic flow can partially or even completely suppress aeroelastic instability. In [17], the suppression mechanisms are investigated numerically. Several aspects of the suppression mechanisms are validated experimentally in [18]. Moreover, an asymptotic analysis is reported in [19] demonstrating the existence of the three passive suppression mechanisms 
based on TET. Suppression of aeroelastic instability of a general nonlinear multi degree of freedom system has also be considered in [20]. Finally, the discussion on relationship between dimensionality of the super-slow manifold, structure of the fixed points and the observed response regimes is explored in review paper [21].

In this context, the use of a NES appears to be an interesting alternative way to control GR instability which contrasts with the use of linear lag dampers having high damping value in order to suppress completely the dynamic instability. For its part, a NES attachment with a relatively small linear damping and a pure nonlinear stiffness, is able to prevent destructive amplitude of oscillations even if GR instability persists. This situations are hereafter referred as partial suppression mechanisms. The goal of the paper is therefore to study the effect of attaching an ungrounded NES on the fuselage of the helicopter for controlling GR instability. A number of the previous cited papers $[14,17,7]$ use numerical methods to analyze the frequency interactions of this kind of essentially nonlinear systems. They demonstrate that high-order resonances between the primary system and the NES may be very significant for adequate understanding transient dynamics in this class of nonlinear systems. These details are beyond the scope of the present paper, since we focus on the characterization of the possible steady-state response regimes of a helicopter ground resonance model including a ungrounded NES attachment assuming a simple 1:1 resonance between the primary system (i.e. the helicopter model) and the NES.

The paper is organized as follows. In Sect. 2, the simplest helicopter model reproducing GR phenomenon is presented. It involves only lag motion of the four blades and one direction of the fuselage motion. Then, a NES is attached to the fuselage in an ungrounded configuration leading to the Simplest Helicopter Model including a NES (hereafter referred as SHM+NES). Preliminary results are presented in Sect. 3 including the linear stability analysis of the trivial solution of the SHM+NES. Moreover, using numerical simulations, the section presents also some steady-state response regimes which result from the NES attachment. We count four regimes classified into two categories depending on the fact that the trivial solution of the SHM+NES is stable or not. In Sect. 4, an analytical procedure based on complexification-averaging method together with geometric singular perturbation theory [22] is developed to analyze situations for which trivial solution of the SHM+NES is unstable. Finally Sect. 5 is dedicated to the prediction of the steady-state response regimes and numerical validation.

\section{System under study}

The system under study consists of a Simplest Helicopter Model (SHM) including a Non Linear Energy Sink (NES). The SHM is first introduced.

\subsection{Simplest Helicopter Model (SHM) that can describe ground resonance}

To carry out the analytical approach presented in this work (in Sect. 4) we need to obtain a mechanical model of a he-

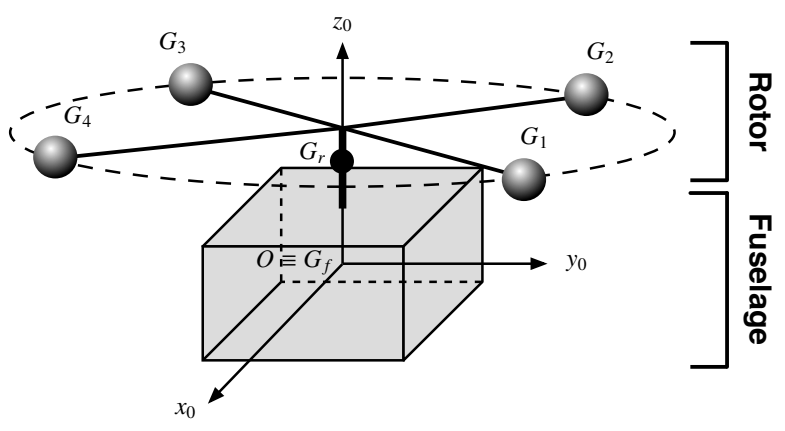

(a)

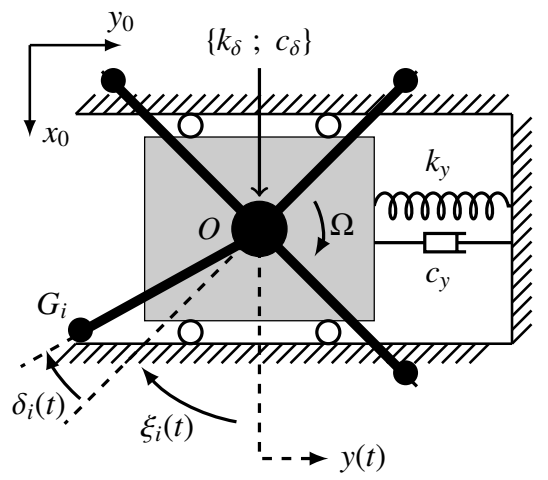

(b)

Figure 1: Descriptive diagram of the used helicopter system. (a) Overview of the system. (b) View from the top.

licopter which can reproduce the ground resonance phenomenon which has the minimum number of degrees of freedom (DOF). For that, a reference helicopter model, with 5 DOF (i.e 10 state variables in state-sapce) is first presented (Section 2.1.1). Next, it is simplified using successively Coleman transformation [1] (Section 2.1.2) and binormal transformation [6] (Section 2.1.3) leading to the SHM which has 4 state variables in state-space.

\subsubsection{Reference model}

The reference model is very similar to that described for example in [2, 3, 4]. Here, it describes an idealized helicopter which consists of a fuselage on which a 4-blades rotor rotates at a constant speed $\Omega$. Moreover, only lag motions are taken into account.

To obtain the equations of motion, a earth-fixed Cartesian coordinate system is considered where the origin, $O$, coincides with the center of inertia $G_{f}$ of the fuselage at rest and the three Cartesian axes, $x_{0^{-}}$axis, $y_{0^{-}}$axis and $z_{0^{-}}$ axis, are shown Fig. 1(a). At rest, the center of inertia of the rotor $G_{r}$ is also located on the $z_{0}$-axis.

The fuselage is a simple damped mass-spring system with only one translational motion alone the $y_{0}$-axis characterized by the coordinate $y(t)$. Each blade is assumed to be a mass point $G_{i}$ (with $i \in[1,4]$ ) placed at a distance $L$ from the $z_{0}$-axis. The position of the $i$ th blade in the $x_{0} y_{0}$-plan is therefore given by 


$$
\left\{\begin{array}{l}
x_{G_{i}}(t)=L \cos \left(\xi_{i}(t)+\delta_{i}(t)\right) \\
y_{G_{i}}(t)=y(t)+L \sin \left(\xi_{i}(t)+\delta_{i}(t)\right),
\end{array}\right.
$$

where $\delta_{i}(t)$ is the lagging angle of the $i$ th blade. The lagging angle is the angle between the current position of the blade and its equilibrium position $\xi_{i}(t)=\Omega t-\frac{\pi}{2}(i-1)$ (see Fig. 1(b)).

The equations of motion which govern the time evolution of the five degrees of freedom of the system (the fuselage displacement $y(t)$ and the four lagging angles $\left.\delta_{i}(t)\right)$ are then derived using Lagrange method. This leads to the reference model

$$
\left\{\begin{array}{l}
\left(m_{y}+4 m_{\delta}\right) \ddot{y}+c_{y} \dot{y}+k_{y} y+ \\
M_{\delta} \sum_{j=1}^{4}\left\{\ddot{\delta}_{j} \cos \left(\xi_{j}+\delta_{j}\right)-\left(\Omega+\dot{\delta}_{j}\right)^{2} \sin \left(\xi_{j}+\delta_{j}\right)\right\} \neq 2 \\
I_{\delta} \ddot{\delta}_{i}+c_{\delta} \dot{\delta}_{i}+k_{\delta} \delta_{i}+M_{\delta} \ddot{y} \cos \left(\xi_{i}+\delta_{i}\right)=0, i=1,4
\end{array}\right.
$$

where " " denotes the derivative with respect to time $t, m_{y}$ is the fuselage mass, $m_{\delta}$ is the one blade mass, $M_{\delta}=m_{\delta} L$ and $I_{\delta}=m_{\delta} L^{2}$ are the static moment and the moment of inertia of one blade respectively, $c_{y}, c_{\delta}$ are damping coefficients of the the fuselage and of a blade respectively and $k_{y}$ and $k_{\delta}$ are linear stiffness coefficients.

To apply Coleman transformation in next section, the reference model (2) must be linearized. To achieve that, assuming small lagging angle motion $\left(\delta_{i}(t)<<1\right)$ around the trivial equilibrium position of the system, Eqs. (2) are expanded in a first-order Taylor series to give the following linear system with periodic coefficients

$$
\left\{\begin{array}{l}
\left(m_{y}+4 m_{\delta}\right) \ddot{y}+c_{y} \dot{y}+k_{y} y+ \\
M_{\delta} \sum_{j=1}^{4}\left\{\left(\ddot{\delta}_{j}-\Omega^{2} \delta_{j}\right) \cos \left(\xi_{j}\right)-2 \Omega \dot{\delta_{j}} \sin \left(\xi_{j}\right)\right\}=0(3 \mathrm{a}) \\
I_{\delta} \ddot{\delta}_{i}+c_{\delta} \dot{\delta}_{i}+k_{\delta} \delta_{i}+M_{\delta} \ddot{y} \cos \left(\xi_{i}\right)=0, i=1,4
\end{array}\right.
$$

\subsubsection{Coleman transformation}

The Coleman transformation ${ }^{1}$ [1] is used to obtain timeinvariant system of equations. It consists in a change of variables which transforms individual motions of the blades (described by the lagging angles) into collective motions described by the so-called Coleman coordinates. For a 4blades rotor there are four Coleman coordinates $\delta_{0}, \delta_{1 c}, \delta_{1 s}$

\footnotetext{
${ }^{1}$ Coleman transformation is sometimes also called multi-blade coordinate transformation.
}

and $\delta_{c p}$ defined by

$$
\begin{aligned}
\delta_{0}(t) & =\frac{1}{4} \sum_{j=1}^{4} \delta_{j}(t) \\
\delta_{1 c}(t) & =\frac{1}{2} \sum_{j=1}^{4} \delta_{j}(t) \cos \left(\xi_{j}(t)\right) \\
\delta_{1 s}(t) & =\frac{1}{2} \sum_{j=1}^{4} \delta_{j}(t) \sin \left(\xi_{j}(t)\right) \\
\delta_{c p}(t) & =\frac{1}{4} \sum_{j=1}^{4}(-1)^{j} \delta_{j}(t) .
\end{aligned}
$$

Considering small lagging angles, it can be shown that the variables $\delta_{1 c}$ and $\delta_{1 s}$ are linked to the position of the center of inertia of the rotor $G_{r}$ in the $x_{0} y_{0}$-plan through

$$
\left\{\begin{array}{l}
x_{G_{r}}(t)=-\frac{L}{2} \delta_{1 s}(t) \\
y_{G_{r}}(t)=\frac{L}{2} \delta_{1 c}(t) .
\end{array}\right.
$$

Using the change of variable (4), Eqs. (3a) and (3b) become respectively

$$
\left(m_{y}+4 m_{\delta}\right) \ddot{y}+c_{y} \dot{y}+k_{y} y+2 M_{\delta} \ddot{\delta}_{1 c}=0,
$$

and,

$$
\left\{\begin{array}{l}
I_{\delta} \ddot{\delta}_{0}+c_{\delta} \dot{\delta}_{0}+k_{\delta} \dot{\delta}_{0}=0 \\
I_{\delta} \ddot{\delta}_{1 c}+c_{\delta} \dot{\delta}_{1 c}+2 I_{\delta} \Omega \dot{\delta}_{1 s}+ \\
\quad\left(k_{\delta}-I_{\delta} \Omega^{2}\right) \delta_{1 c}+c_{\delta} \Omega \delta_{1 s}+M_{\delta} \ddot{y}=0 \\
I_{\delta} \ddot{\delta}_{1 s}+c_{\delta} \dot{\delta}_{1 s}-2 I_{\delta} \Omega \dot{\delta}_{1 c}+ \\
\quad\left(k_{\delta}-I_{\delta} \Omega^{2}\right) \delta_{1 s}-c_{\delta} \Omega \delta_{1 c}=0 \\
I_{\delta} \ddot{\delta}_{c p}+c_{\delta} \dot{\delta}_{c p}+k_{\delta} \dot{\delta}_{c p}=0 .
\end{array}\right.
$$

Eqs. (7a) and (7d) show that the variables $\delta_{0}$ and $\delta_{c p}$ are uncoupled and can be discarded. The reason of the decoupling is the fact that the collective motions $\delta_{0}$ and $\delta_{c p}$ leave the rotor center of inertia motionless. As a result, a system of equations with three DOF, namely $y, \delta_{1 c}$ and $\delta_{1 s}$, is obtained.

Introducing the following notations

$$
\begin{aligned}
& {\underset{\sim}{\omega}}_{y}^{2}=k_{y} /\left(m_{y}+4 m_{\delta}\right), \quad \tilde{\tilde{\lambda}}_{\delta}^{2}=k_{\delta} / I_{\delta} ; \\
& \tilde{\lambda}_{y}=c_{y} /\left(m_{y}+4 m_{\delta}\right), \quad \tilde{\lambda}_{\delta}=c_{\delta} / I_{\delta} \\
& \tilde{S}_{d}=2 M_{\delta} /\left(m_{y}+4 m_{\delta}\right), \quad \tilde{S}_{c}=M_{\delta} / I_{\delta}=1 / L .
\end{aligned}
$$

where, $\omega_{y}$ and $\omega_{\delta}$ are the natural frequency of the fuselage and of one blade respectively, $\tilde{S}_{c}$ and $\tilde{S}_{d}$ are the coupling coefficients, equations of motion are finally written in matrix form

$$
\mathbf{M} \ddot{\mathbf{X}}+(\mathbf{C}+\mathbf{G}) \dot{\mathbf{X}}+\mathbf{K X}=\mathbf{0}
$$




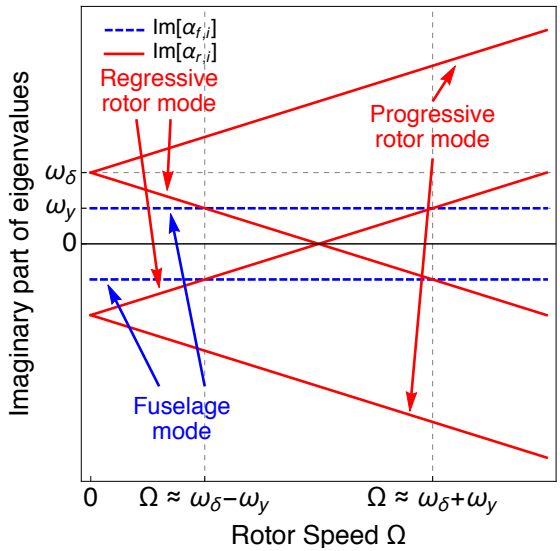

(a)

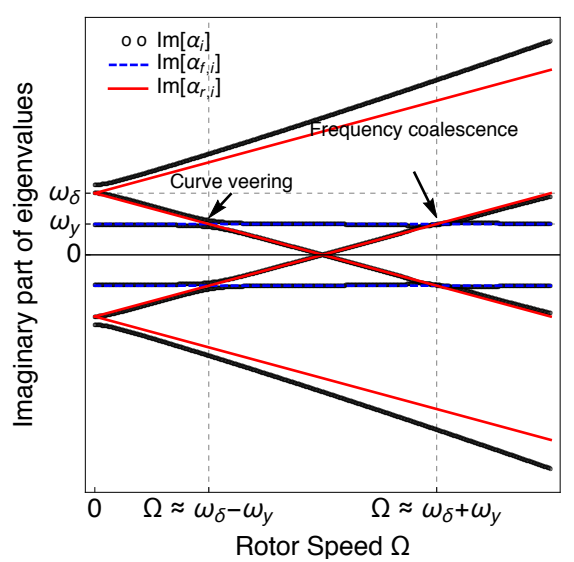

(b)

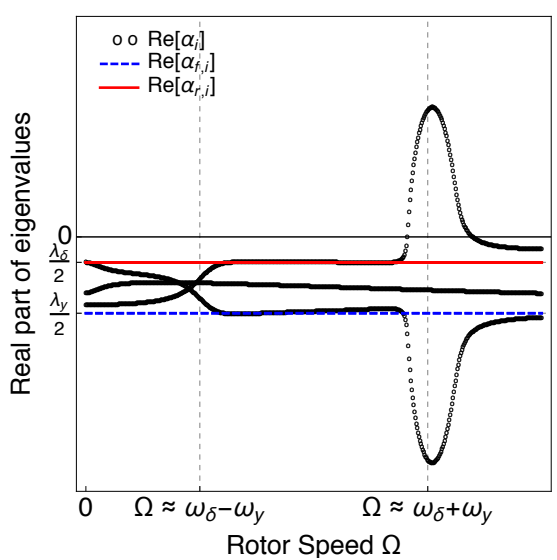

(c)

Figure 2: Eigenvalues of the uncoupled system and coupled system (9) for $\omega_{y}<\omega_{\delta}$. (a) Imaginary parts of the natural eigenvalues $\alpha_{f}$ of the fuselage (dashed blue line) and $\alpha_{r}$ of the rotor (solid red line). Comparison between the eigenvalues of the uncoupled systems (i.e. $\alpha_{f}$ and $\alpha_{r}$ ) and the eigenvalues $\alpha$ of the coupled system (black circles): (b) imaginary parts and (c) real parts. Parameters used: $\omega_{y}=1, \omega_{\delta}=2, \tilde{\lambda}_{y}=0.09, \tilde{\lambda}_{\delta}=0.03, \tilde{S}_{c}=0.6$ and $\tilde{S}_{d}=0.3$. The parameters $\tilde{\lambda}_{y}, \tilde{\lambda}_{\delta}, \tilde{S}_{c}$ and $\tilde{S}_{d}$ are chosen to obtain readable figures, no for their realism.

with,

$$
\mathbf{X}=\left[\begin{array}{lll}
y & \delta_{1 c} & \delta_{1 s}
\end{array}\right]^{t} .
$$

$\mathbf{M}, \mathbf{K}, \mathbf{C}$ and $\mathbf{G}$, are mass matrix, stiffness matrix, damping matrix and gyroscopic matrix of the system respectively, they are defined by

$$
\begin{array}{ll}
\mathbf{M}=\left[\begin{array}{ccc}
1 & \tilde{S}_{d} & 0 \\
\tilde{S}_{c} & 1 & 0 \\
0 & 0 & 1
\end{array}\right], & \mathbf{K}=\left[\begin{array}{ccc}
\omega_{y}^{2} & 0 & 0 \\
0 & \omega_{\delta}^{2}-\Omega^{2} & \tilde{\lambda}_{\delta} \Omega \\
0 & -\tilde{\lambda}_{\delta} \Omega & \omega_{\delta}^{2}-\Omega^{2}
\end{array}\right] \\
\mathbf{C}=\left[\begin{array}{ccc}
\tilde{\lambda}_{y} & 0 & 0 \\
0 & \tilde{\lambda}_{\delta} & 0 \\
0 & 0 & \tilde{\lambda}_{\delta}
\end{array}\right], \quad \mathbf{G}=\left[\begin{array}{ccc}
0 & 0 & 0 \\
0 & 0 & 2 \Omega \\
0 & -2 \Omega & 0
\end{array}\right] .
\end{array}
$$

Note that $\tilde{S}_{d}$ and $\tilde{S}_{c}$ characterize the fuselage/rotor coupling.

Helicopter ground resonance. The phenomenon is analyzed making a stability analysis of the previous linear system (9). For that, the equations of motion are written using a state-space form

$$
\dot{\mathbf{U}}=\mathbf{A} \mathbf{U}
$$

with,

$$
\mathbf{U}=\left[\begin{array}{llllll}
y & \delta_{1 c} & \delta_{1 s} & \dot{y} & \dot{\delta}_{1 c} & \dot{\delta}_{1 s}
\end{array}\right]^{t},
$$

and,

$$
\mathbf{A}=\left[\begin{array}{c|c}
\mathbf{0} & \mathbf{I} \\
\hline-\mathbf{M}^{-1} \mathbf{K} & -\mathbf{M}^{-1}(\mathbf{C}+\mathbf{G}) .
\end{array}\right] .
$$

Then the set of eigenvalues $\alpha_{i}$ (with $i \in[1,6]$ ) of the matrix $\mathbf{A}$ are computed.

If the fuselage/rotor coupling is suppressed (i.e. stating $\left.\tilde{S}_{c}=\tilde{S}_{d}=0\right)$, the eigenvalues of the system correspond to the natural eigenvalues of the fuselage, denoted $\alpha_{f, i}$ (with $i \in[1,2]$ ), and of the rotor, denoted $\alpha_{r, i}$ (with $i \in[1,4]$ ). In Fig. 2(a), the typical behavior of the imaginary part of these eigenvalues is reported with respect to the rotor speed $\Omega$ for $\omega_{y}<\omega_{\delta}$. We can notice that there are two values of $\Omega$ for which an interaction between the fuselage mode and the regressive rotor mode is possible: $\Omega \approx\left|\omega_{y}-\omega_{\delta}\right|$ and $\Omega \approx \omega_{y}+\omega_{\delta}^{2}$.

In Figs. 2(b) and 2(c), the comparison between eigenvalues of the uncoupled systems $\alpha_{f, i}$ and $\alpha_{r, i}$ and the eigenvalues $\alpha_{i}$ of the coupled system shows that:

- For $\Omega \approx\left|\omega_{y}-\omega_{\delta}\right|$, a phenomenon of "curve veering" [23, 24] appears, the real part of the eigenvalues $\alpha$ stay negative and there is no instability.

- For $\Omega \approx \omega_{y}+\omega_{\delta}$, a phenomenon of frequency coalescence is observed, the real part of one of the eigenvalues $\alpha$ becomes positive and a dynamic instability occurs; this is the helicopter ground resonance.

Fig. 2 shows also that the progressive rotor mode does not interact with the fuselage mode.

A situation with $\omega_{y}>\omega_{\delta}$ can also be encountered leading to a possible interaction between the fuselage mode and the progressive rotor mode at $\Omega \approx\left|\omega_{y}-\omega_{\delta}\right|$. However, a similar plot to that of Fig. 2 with $\omega_{y}>\omega_{\delta}$ shows that this interaction produces a "curve veering" phenomenon. Consequently, in both situations $\left(\omega_{y}<\omega_{\delta}\right.$ and $\left.\omega_{y}>\omega_{\delta}\right)$ the progressive rotor mode does not contribute to the creation of the GR instability. Therefore, the last step to obtain the simplest model for helicopter ground resonance is to eliminate the progressive rotor mode from the equations of motion. This is achieved in the following section using bi-normal transformation.

\footnotetext{
${ }^{2}$ If undamped system would be considered (i.e. if $\lambda_{y}=\lambda_{\delta}=0$ ), we would get exact equalities: $\Omega=\left|\omega_{y}-\omega_{\delta}\right|$ and $\Omega=\omega_{y}+\omega_{\delta}$, see [2] for more details.
} 


\subsubsection{Diagonalization of the rotor equations of motion: bi-} normal transformation

Equations of motion of the rotor alone are

$$
\mathbf{M}_{\mathbf{r}} \ddot{\mathbf{X}}_{\mathbf{r}}+\left(\mathbf{C}_{\mathbf{r}}+\mathbf{G}_{\mathbf{r}}\right) \dot{\mathbf{X}}_{\mathbf{r}}+\mathbf{X}_{\mathbf{r}} \mathbf{K}_{\mathbf{r}}=\mathbf{0}
$$

with,

$$
\mathbf{X}_{\mathbf{r}}=\left[\begin{array}{ll}
\delta_{1 c} & \delta_{1 s}
\end{array}\right]^{t}
$$

and,

$$
\begin{aligned}
\mathbf{M}_{\mathbf{r}}=\left[\begin{array}{ll}
1 & 0 \\
0 & 1
\end{array}\right], & \mathbf{K}_{\mathbf{r}}=\left[\begin{array}{cc}
\omega_{\delta}^{2}-\Omega^{2} & \tilde{\lambda}_{\delta} \Omega \\
-\tilde{\lambda}_{\delta} \Omega & \omega_{\delta}^{2}-\Omega^{2}
\end{array}\right], \\
\mathbf{C}_{\mathbf{r}}=\left[\begin{array}{cc}
\tilde{\lambda}_{\delta} & 0 \\
0 & \tilde{\lambda}_{\delta}
\end{array}\right], & \mathbf{G}_{\mathbf{r}}=\left[\begin{array}{cc}
0 & 2 \Omega \\
-2 \Omega & 0
\end{array}\right] .
\end{aligned}
$$

Due to the presence of the gyroscopic matrix $\mathbf{G}_{\mathbf{r}}$, the diagonalization of the system of Eqs. (16) must necessarily be carried out in state-space using binormal transformation. The general procedure of the binormal transformation is provided for example in $[25,26]$. In the context of the helicopter modeling the binormal transformation was introduced by Done [6]. The method is briefly recalled in this section.

In state-space form, equations of motion (16) are written as follows

$$
\dot{\mathbf{U}}_{\mathbf{r}}=\mathbf{A}_{\mathbf{r}} \mathbf{U}_{\mathbf{r}}
$$

with,

$$
\mathbf{U}_{\mathbf{r}}=\left[\begin{array}{llll}
\delta_{1 c} & \delta_{1 s} & \dot{\delta}_{1 c} & \dot{\delta}_{1 s}
\end{array}\right]^{t},
$$

and,

$$
\mathbf{A}_{\mathbf{r}}=\left[\begin{array}{c|c}
\mathbf{0} & \mathbf{I} \\
\hline-\mathbf{M}_{\mathbf{r}}^{-1} \mathbf{K}_{\mathbf{r}} & -\mathbf{M}_{\mathbf{r}}^{-\mathbf{1}}\left(\mathbf{C}_{\mathbf{r}}+\mathbf{G}_{\mathbf{r}}\right)
\end{array}\right] .
$$

The following eigenvalue problems:

$$
\mathbf{A}_{\mathbf{r}} \mathbf{r}=\alpha_{\mathbf{r}} \mathbf{r} \quad \text { and } \quad \mathbf{A}_{\mathbf{r}}^{\mathbf{t}} \mathbf{l}=\alpha_{\mathbf{r}} \mathbf{l}
$$

where $\mathbf{A}_{\mathbf{r}}^{\mathbf{t}}$ denotes the transpose of $\mathbf{A}_{\mathbf{r}}$, are solved giving:

- two pairs of complex conjugates eigenvalues: $\alpha_{r, 1}, \alpha_{r, 1}^{*}$, $\alpha_{r, 2}$ and $\alpha_{r, 2}^{*}$ (the " * " is the usual notation for the complex conjugate),

- two pairs of complex conjugates eigenvectors of $\mathbf{A}_{\mathbf{r}}, \mathbf{r}_{\mathbf{i}}$, called right eigenvectors of $\mathbf{A}_{\mathbf{r}}: \mathbf{r}_{\mathbf{1}}, \mathbf{r}_{\mathbf{1}}^{*}, \mathbf{r}_{\mathbf{2}}$ and $\mathbf{r}_{\mathbf{2}}^{*}$.

- two pairs of complex conjugates eigenvectors of $\mathbf{A}_{\mathbf{r}}^{\mathbf{t}}, \mathbf{l}_{\mathbf{i}}$, called left eigenvectors of $\mathbf{A}_{\mathbf{r}}$ : $\mathbf{l}_{\mathbf{1}}, \mathbf{l}_{\mathbf{1}}^{*}, \mathbf{l}_{\mathbf{2}}$ and $\mathbf{l}_{\mathbf{2}}^{*}$.

The right and left eigenvectors satisfy the biorthogonality properties: $\mathbf{L}^{\mathbf{t}} \mathbf{R}$ and $\mathbf{L}^{\mathbf{t}} \mathbf{A}_{\mathbf{r}} \mathbf{R}$ are diagonal matrices where $\mathbf{R}=\left[\begin{array}{llll}\mathbf{r}_{\mathbf{1}} & \mathbf{r}_{\mathbf{1}}^{*} & \mathbf{r}_{\mathbf{2}} & \mathbf{r}_{\mathbf{2}}^{*}\end{array}\right]$ and $\mathbf{L}=\left[\begin{array}{llll}\mathbf{l}_{\mathbf{1}} & \mathbf{l}_{\mathbf{1}}^{*} & \mathbf{l}_{\mathbf{2}} & \mathbf{l}_{\mathbf{2}}^{*}\end{array}\right]$. It is convenient to normalize the two set of eigenvectors $\mathbf{r}_{\mathbf{i}}$ and $\mathbf{l}_{\mathbf{i}}$ in order to obtain

$$
\mathbf{L}^{\mathbf{t}} \mathbf{R}=\mathbf{I}
$$

In this case, we have:

$$
\mathbf{L}^{\mathbf{t}} \mathbf{A}_{\mathbf{r}} \mathbf{R}=\left[\begin{array}{cccc}
\alpha_{r, 1} & 0 & 0 & 0 \\
0 & \alpha_{r, 1^{*}} & 0 & 0 \\
0 & 0 & \alpha_{r, 2} & 0 \\
0 & 0 & 0 & \alpha_{r, 2^{*}}
\end{array}\right]=\mathbf{D}_{\mathbf{r}}
$$

The binormal transformation consists in introducing the binormal coordinates which are constituted of two pairs of complex conjugates, $\left(q_{1}, q_{1}^{*}\right)$ and $\left(q_{2}, q_{2}^{*}\right)$, and defined by the following relation

$$
\mathbf{U}_{\mathbf{r}}=\mathbf{R Q} \Leftrightarrow \mathbf{Q}=\mathbf{L}^{\mathbf{t}} \mathbf{U}_{\mathbf{r}}, \quad \text { with } \quad \mathbf{Q}=\left[\begin{array}{lll}
q_{1} q_{1}^{*} q_{2} & q_{2}^{*}
\end{array}\right]^{t} .
$$

From Eqs. (5), the binormal coordinates can be interpreted as linear combinations, with complex coefficients, of the position and the velocity of the center of inertia of the rotor $G_{r}$. Introducing Eq. (26) in Eq. (20), the equations of motion of the rotor take the form of the following diagonal system

$$
\dot{\mathrm{Q}}=\mathbf{D}_{\mathbf{r}} \mathbf{Q} \text {. }
$$

One of the couples $\left(q_{1}, q_{1}^{*}\right)$ and $\left(q_{2}, q_{2}^{*}\right)$ is relative to the progressive rotor mode and the other the regressive one. The couple $\left(q_{2}, q_{2}^{*}\right)$ is arbitrary chosen to be relative to the progressive rotor mode and since this mode does not destabilize the system, the variables $q_{2}$ and $q_{2}^{*}$ can be removed from the analysis.

Consequently, using the vector $\mathbf{U}_{\mathbf{r}}$, equations of motion of the whole coupled system, i.e. Eqs (9), become

$$
\left\{\begin{array}{c}
\ddot{y}+\tilde{\lambda}_{y} \dot{y}+\omega_{y}^{2} y+\tilde{S}_{d} \dot{\mathbf{U}}_{\mathbf{r}, 3}=0 \\
\dot{\mathbf{U}}_{\mathbf{r}}=\mathbf{A}_{\mathbf{r}} \mathbf{U}_{\mathbf{r}}+\left[\begin{array}{c}
0 \\
0 \\
\tilde{S}_{c} \ddot{y} \\
0
\end{array}\right]
\end{array}\right.
$$

Then using Eq. (27) (ignoring variables $q_{2}$ and $q_{2}^{*}$ ) and the following relations

$$
\alpha_{r, 2}=\alpha_{r, 1}^{*}, \quad \mathbf{R}_{32}=\mathbf{R}_{31}^{*}, \quad \mathbf{L}_{32}=\mathbf{L}_{31}^{*},
$$

Eqs. (28) become finally

$$
\left\{\begin{array}{l}
\ddot{y}+\tilde{\lambda}_{y} \dot{y}+\omega_{y}^{2} y+\tilde{S}_{d}\left(\mathbf{R}_{31} \dot{q}_{1}+\mathbf{R}_{31}^{*} \dot{q}_{1}^{*}\right)=0 \\
\dot{q}_{1}-\alpha_{r, 1} q_{1}+\tilde{S}_{c} \mathbf{L}_{31} \ddot{y}=0 .
\end{array}\right.
$$

Eqs. (30) define the Simplest Helicopter Model (the SHM with 4 state variables in state-space) that can describe GR phenomenon.

\subsection{The Simplest Helicopter Model including a Non Linear Energy Sink (SHM+NES)}

The simplified model (30) is used to study the effect of attaching a NES on the fuselage of the helicopter. For that, a NES with a mass $m_{h}$, a damping coefficient $c_{h}$ and a cubic 


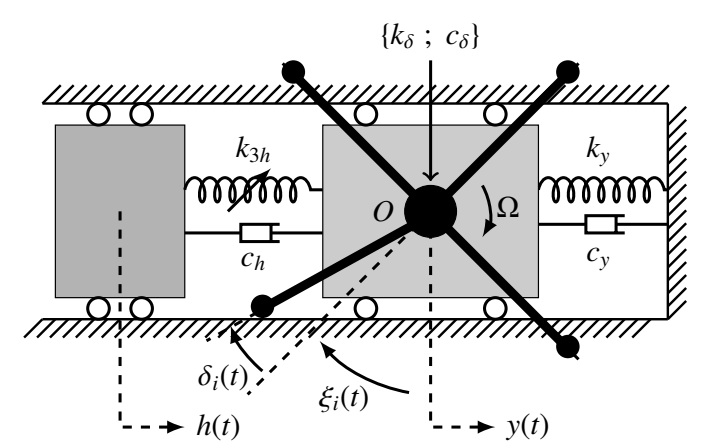

Figure 3: Descriptive diagram of the used helicopter system coupled to an ungrounded NES. View from the top.

stiffness $k_{3 h}$, is attached on the fuselage in an ungrounded configuration (see Fig. 3). Taking into account the NES displacement $h(t)$, the equations of motion become

$$
\left\{\begin{array}{c}
\ddot{y}+\tilde{\lambda}_{y} \dot{y}+\omega_{y}^{2} y+\tilde{S}_{d}\left(\mathbf{R}_{31} \dot{q}_{1}+\mathbf{R}_{31}^{*} \dot{q}_{1}^{*}\right)+ \\
\tilde{\mu}(\dot{y}-\dot{h})+\tilde{\alpha}_{3}(y-h)^{3}=0 \\
\epsilon \ddot{h}+\tilde{\mu}(\dot{h}-\dot{y})+\tilde{\alpha}_{3}(h-y)^{3}=0 \\
\dot{q}_{1}-\alpha_{r, 1} q_{1}+\tilde{S}_{c} \mathbf{L}_{31} \ddot{y}=0,
\end{array}\right.
$$

where $\epsilon=m_{h} /\left(m_{y}+4 m_{\delta}\right)$ is the mass ratio, $\tilde{\mu}=$ $c_{h} /\left(m_{y}+4 m_{\delta}\right)$ and $\tilde{\alpha}_{3}=k_{3 h} /\left(m_{y}+4 m_{\delta}\right)$.

System of Eqs. (31) is the Simplest Helicopter Model including a Non Linear Energy Sink (SHM+NES). The remaining of the paper is devoted to the analysis of its steadystate regimes.

\section{Steady-state response regimes of the SHM+NES: preliminary results}

\subsection{Fixed points of the SHM+NES and their sta- bility}

It is easy to show that the only fixed point of the SHM+NES (Eqs (31)) is the trivial solution $y=h=q_{1}=$ 0 . To find its stability, the 6 eigenvalues of the Jacobian matrix of the state-space representation of the system Eqs (31) have to be computed. The trivial solution is unstable is one of the eigenvalues have positive real part.

\subsection{Some steady-state response regimes}

The aim of this section is first to present the steady-state response regimes which may result from the NES attachment and its relevance. For that, the time series $y(t)$, resulting from the numerical integration of the SHM+NES, Eqs. (31), and the reference model ${ }^{3}$, Eqs. (2), are compared in Fig. 4. In both cases, same initial conditions are used, chosen as small perturbation of the trivial solution: $y(0)=0.1, h(0)=\dot{y}(0)=\dot{h}(0)=q_{1}(0)=0$.

\footnotetext{
${ }^{3}$ The reference model does not include NES attachment but saturation mechanisms can appear due to the nonlinear nature of this model.
}

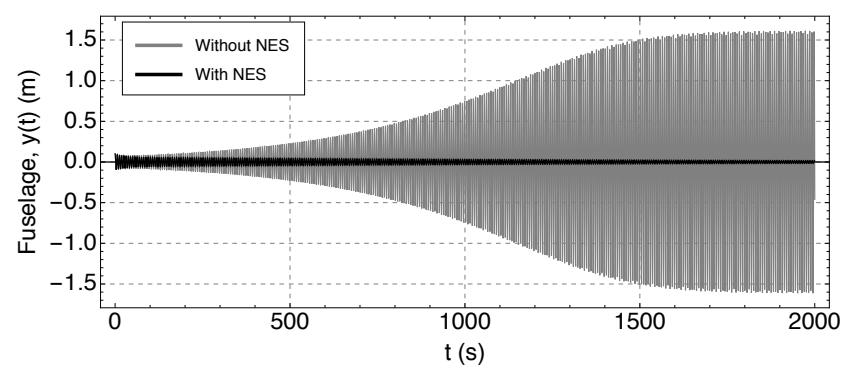

(a) Complete suppression

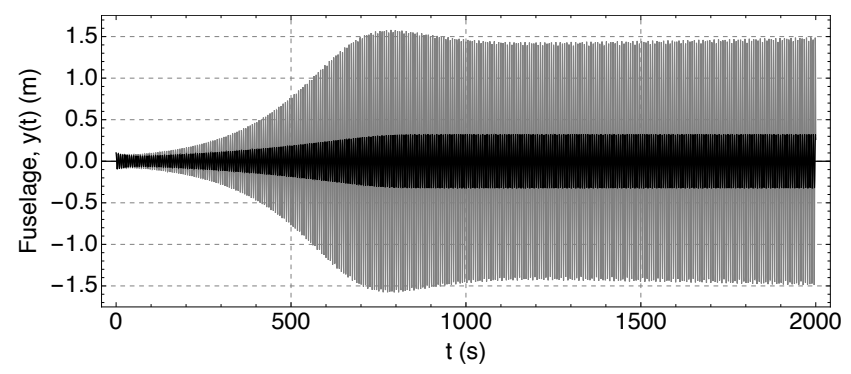

(b) Partial suppression: PR

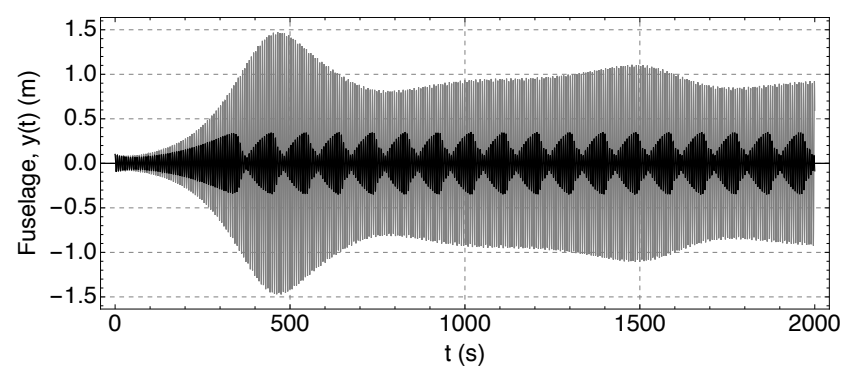

(c) Partial suppression: SMR

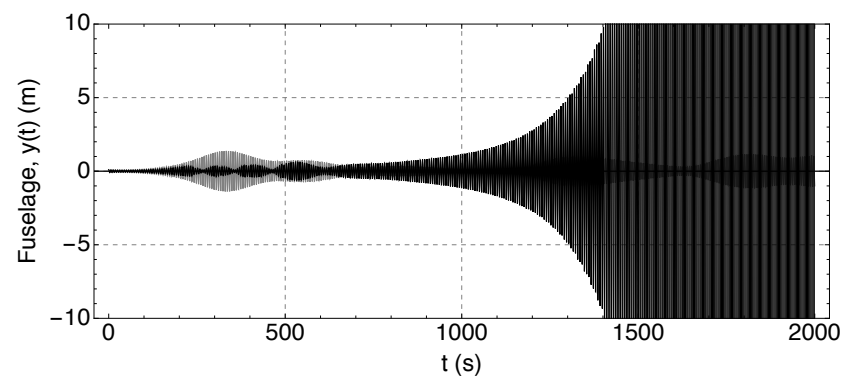

(d) No suppression

Figure 4: Comparison between time series $y(t)$ resulting from the numerical integration of the system of Eqs. (31) (solid black line) and $y(t)$ result of the numerical integration of the reference model (Eqs. (2)) (solid gray line). The following set of parameters is used: $\omega_{y}=1, \omega_{\delta}=2, \Omega=\omega_{y}+\omega_{\delta}, \tilde{\lambda}_{y}=0.02, \tilde{S}_{c}=0.1$, $\tilde{S}_{d}=0.05, \alpha_{3}=0.1$ and $\mu=0.01$ and $\epsilon=0.05$. (a) $\tilde{\lambda}_{\delta}=0.045$, (b) $\tilde{\lambda}_{\delta}=0.03$, (c) $\tilde{\lambda}_{\delta}=0.015$ and (d) $\tilde{\lambda}_{\delta}=0.0005$.

Observing the fuselage displacement $y(t)$ (solid black line in Fig. 4) of the SHM+NES, the four different types of response regimes which may be generated when a NES is attached on the fuselage are highlighted selecting different values of the blade damping $\tilde{\lambda}_{\delta}$. They are classified into two categories depending on the fact that the trivial solution of the SHM+NES is stable or not:

- The trivial solution of the SHM+NES is stable: 
$\Rightarrow$ Complete suppression (see. Fig. 4(a)). In this case, the additional damping due to the NES attachment stabilizes the system and the GR instability is completely suppressed.

\section{- The trivial solution of the SHM+NES is unstable:}

$\Rightarrow$ Partial suppression through Periodic Response (PR) (see. Fig. 4(b)). In this case, the steady-state response regime is periodic with frequency close to $\omega_{y}{ }^{4}$.

$\Rightarrow$ Partial suppression through Strongly Modulated Response (SMR) (see. Fig. 4(c)). In this case, the steady-state response regime is a quasiperiodic regime which exhibits a "fast" component with frequency close to $\omega_{y}$ and a "slow" component corresponding to the envelope of the signal. The term "Strongly modulated response" has been introduced by Starosvetsky and Gendelman [10] for the study of a forced linear system coupled to a NES.

$\Rightarrow$ No suppression of GR (see. Fig. $4(d)$ ). The NES is not able to maintain stable steady-state regimes. We observe exponential growth of the fuselage displacement.

These four responses are also observed by Lee et al. [17] and study theoretically by Gendelman et al. [19] in the context of the control of aeroelastic instabilities of a rigid wing in subsonic flow by means of a NES.

In the following section an analytical procedure based on complexification-averaging method together with geometric singular perturbation theory is developed to analyze situations for which trivial solution of the SHM+NES is unstable.

\section{Steady-state response regimes of the SHM+NES: theoretical study}

\subsection{The complexified-averaged model}

The analytical study proposed is based on complexificationaveraging method first introduced by Manevitch [11] and discussed in detail by Vakakis et al. [7].

First, to simplify the following calculations, it is convenient to introduce barycentric coordinates $v(t)$ and $w(t)$

$$
v=y+\epsilon h \quad \text { and } \quad w=y-h,
$$

and reciprocally,

$$
y=\frac{v+\epsilon w}{\epsilon+1} \quad \text { and } \quad h=\frac{v-w}{\epsilon+1} .
$$

Using Eqs. (32) and (33), Eqs. (31) are written as follow

\footnotetext{
${ }^{4}$ This can be shown for example by computing the power spectrum of the steady part of the signal.
}

$$
\left\{\begin{array}{c}
\ddot{v}+\tilde{\lambda}_{y} \frac{\dot{v}+\epsilon \dot{w}}{\epsilon+1}+\omega_{y}^{2} \frac{v+\epsilon w}{\epsilon+1}+\tilde{S}_{d}\left(\mathbf{R}_{31} \dot{q}_{1}+\mathbf{R}_{31}^{*} \dot{q}_{1}^{*}\right)(34 \mathrm{z}) \\
\ddot{w}+\tilde{\lambda}_{y} \frac{\dot{v}+\epsilon \dot{w}}{\epsilon+1}+\omega_{y}^{2} \frac{v+\epsilon w}{\epsilon+1}+\tilde{S}_{d}\left(\mathbf{R}_{31} \dot{q}_{1}+\mathbf{R}_{31}^{*} \dot{q}_{1}^{*}\right)+ \\
\tilde{\mu} \frac{1+\epsilon}{\epsilon} \dot{w}+\tilde{\alpha}_{3} \frac{1+\epsilon}{\epsilon} w^{3}=0 \\
\dot{q}_{1}-\alpha_{r, 1} q_{1}+\tilde{S}_{c} \mathbf{L}_{31} \frac{\ddot{v}+\epsilon \ddot{w}}{\epsilon+1}=0 .
\end{array}\right.
$$

Secondly, the complexification ${ }^{5}$ consists in introducing the following change of variable

$$
\psi_{1}=\dot{v}+j \omega_{y} v \quad \text { and } \quad \psi_{2}=\dot{w}+j \omega_{y} w,
$$

with $j^{2}=-1$. Then, the variable $v$ and $w$ are expressed in term of the new variables $\psi_{i}$ as:

$v=\frac{\psi_{1}-\psi_{1}^{*}}{2 j \omega_{y}}, \quad \dot{v}=\frac{\psi_{1}+\psi_{1}^{*}}{2} \quad$ and $\quad \ddot{v}=\dot{\psi}_{1}-\frac{j \omega_{y}}{2}\left(\psi_{1}+\psi_{1}^{*}\right)$,

$w=\frac{\psi_{2}-\psi_{2}^{*}}{2 j \omega_{y}}, \quad \dot{w}=\frac{\psi_{2}+\psi_{2}^{*}}{2} \quad$ and $\quad \ddot{w}=\dot{\psi}_{2}-\frac{j \omega_{y}}{2}\left(\psi_{2}+\psi_{2}^{*}\right)$.

Previous numerical and theoretical results (see Sect. 3.2) motive us to introduce the assumption that the variable $v$, $w$ and $q_{1}$ may be broken down into fast and slow components. For that, the following representation is introduced

$$
\psi_{1}=\phi_{1} e^{j \omega_{y} t}, \quad \psi_{2}=\phi_{2} e^{j \omega_{y} t}, \quad q_{1}=\phi_{3} e^{j \omega_{y} t},
$$

where $\phi_{i}$ (with $i \in[1,3]$ ) is the complex slow modulated amplitude of the fast component $e^{j \omega_{y} t}$.

Substituting Eqs. (36) and (37) into Eqs. (34) an equivalent complex system of differential equations is obtained. Substituting next Eq. (38) in this complex system and performing an averaging over one period of the frequency $\omega_{y}$ yield to a system of equations describing the behavior of the slow complex amplitudes $\phi_{i}$

\footnotetext{
${ }^{5}$ This step is not necessary for the variable $q_{1}(t)$ because it is already a complex variable.
} 


$$
\left\{\begin{array}{c}
\dot{\phi}_{1}+j \frac{\omega_{y}}{2} \phi_{1}+\frac{\tilde{\lambda}_{y}-j \omega_{y}}{2(1+\epsilon)}\left(\phi_{1}+\epsilon \phi_{2}\right)+ \\
\tilde{S}_{d} \mathbf{R}_{31}\left(\dot{\phi}_{3}+j \omega_{y} \phi_{3}\right)=0 \\
\dot{\phi}_{2}+j \frac{\omega_{y}}{2} \phi_{2}+\frac{\tilde{\lambda}_{y}-j \omega_{y}}{2(1+\epsilon)}\left(\phi_{1}+\epsilon \phi_{2}\right)+ \\
\tilde{S}_{d} \mathbf{R}_{31}\left(\dot{\phi}_{3}+j \omega_{y} \phi_{3}\right)+ \\
\frac{\tilde{\mu}}{2} \frac{1+\epsilon}{\epsilon} \phi_{2}-j \frac{3 \tilde{\alpha}_{3}}{8 \omega_{y}^{3}} \frac{1+\epsilon}{\epsilon} \phi_{2}\left|\phi_{2}\right|^{2}=0 \quad(39 \mathrm{~b}) \\
\dot{\phi}_{3}+\left(j \omega_{y}-\alpha_{r, 1}\right) \phi_{3}+ \\
\left.\frac{\tilde{S}_{c}}{1+\epsilon} \mathbf{L}_{31}\left[\left(\dot{\phi}_{1}+\epsilon \dot{\phi}_{2}\right)+j \frac{\omega_{y}}{2}\left(\phi_{1}+\epsilon \phi_{2}\right)\right]=q 39 \mathrm{c}\right)
\end{array}\right.
$$

Eqs. (39) define the complexified-averaged system.

\subsection{Approximation of the periodic solutions of the SHM+NES and their stability}

The trivial fixed point is common to both the non-averaged system (Eq. (31) or (34)) and the complexified-averaged system (39). The stability of this trivial fixed point is calculated in Sect. 3.1 directly on the SHM+NES.

On the other hand, the nontrivial fixed point of the complexified-averaged system (39) (defined as $\dot{\phi}_{i}=0$ for $i \in[1,3]$ ) only characterizes periodic solutions of Eqs. (34) if the frequency of the periodic solutions is equal to $\omega_{y}$, the frequency used to defined the complex variables (35). However, using the polar coordinates $n_{i}(t)$ and $\theta_{i}(t)$ (with $i \in[1,3])$, defined by

$$
\phi_{i}(t)=n_{i}(t) e^{j \theta_{i}(t)},
$$

and considering not the arguments $\theta_{i}(t)$ directly but the argument differences $\delta_{i 1}=\theta_{i}(t)-\theta_{1}(t)$, the periodic solutions of the system of Eqs. (34) (and consequently of the SHM+NES (31)) may be defined from the complexifiedaveraged system (39) as the nontrivial fixed points of the system of differential equations describing the dynamic of the variables $n_{1}, n_{2}, n_{3}, \delta_{21}$ and $\delta_{31}$. See A for more details.

To obtain this system, Eqs. (39) are first re-written using matrix form

$$
\dot{\boldsymbol{\Phi}}=\mathbf{C} \boldsymbol{\Phi}+\phi_{2}\left|\phi_{2}\right|^{2} \mathbf{H},
$$

where the constant complex matrices $\mathbf{C}$ and $\mathbf{H}$ are not specified (and easy obtained from Eqs. (39)).

Next introducing the polar coordinates (40) and separating real and imaginary parts of each equation, Eq. (41) take the form

$$
\left\{\begin{array}{c}
\dot{n}_{1}=n_{1} \operatorname{Re}\left[\mathbf{C}_{11}\right]+n_{2} \operatorname{Re}\left[\mathbf{C}_{12} e^{j \delta_{21}}\right]+ \\
n_{3} \operatorname{Re}\left[\mathbf{C}_{13} e^{j \delta_{31}}\right]+n_{2}^{3} \operatorname{Re}\left[\mathbf{H}_{1} e^{j \delta_{21}}\right] \\
n_{1} \dot{\theta}_{1}=n_{1} \operatorname{Im}\left[\mathbf{C}_{11}\right]+n_{2} \operatorname{Im}\left[\mathbf{C}_{12} e^{j \delta_{21}}\right]+ \\
n_{3} \operatorname{Im}\left[\mathbf{C}_{13} e^{j \delta_{31}}\right]+n_{2}^{3} \operatorname{Im}\left[\mathbf{H}_{1} e^{j \delta_{21}}\right] \\
\dot{n}_{2}=n_{1} \operatorname{Re}\left[\mathbf{C}_{21} e^{-j \delta_{21}}\right]+ \\
n_{2} \operatorname{Re}\left[\mathbf{C}_{22}\right]+n_{3} \operatorname{Re}\left[\mathbf{C}_{23} e^{j\left(\delta_{31}-\delta_{21}\right)}\right]+n_{2}^{3} \operatorname{Re}\left[\mathbf{H}_{2}\right](42 \mathrm{c}) \\
n_{2} \dot{\theta}_{2}=n_{1} \operatorname{Im}\left[\mathbf{C}_{21} e^{-j \delta_{21}}\right]+ \\
n_{2} \operatorname{Im}\left[\mathbf{C}_{22}\right]+n_{3} \operatorname{Im}\left[\mathbf{C}_{23} e^{j\left(\delta_{31}-\delta_{21}\right)}\right]+n_{2}^{3} \operatorname{Im}\left[\mathbf{H}_{2}(42 \mathrm{~d})\right. \\
\dot{n}_{3}=n_{1} \operatorname{Re}\left[\mathbf{C}_{31} e^{-j \delta_{31}}\right]+n_{2} \operatorname{Re}\left[\mathbf{C}_{32} e^{j\left(\delta_{21}-\delta_{31}\right)}\right]+ \\
n_{3} \operatorname{Re}\left[\mathbf{C}_{33}\right]+n_{2}^{3} \operatorname{Re}\left[\mathbf{H}_{3} e^{j\left(\delta_{21}-\delta_{31}\right)}\right] \\
n_{3} \dot{\theta}_{3}=n_{1} \operatorname{Im}\left[\mathbf{C}_{31} e^{-j \delta_{31}}\right]+n_{2} \operatorname{Im}\left[\mathbf{C}_{32} e^{j\left(\delta_{21}-\delta_{31}\right)}\right]+ \\
n_{3} \operatorname{Im}\left[\mathbf{C}_{33}\right]+n_{2}^{3} \operatorname{Im}\left[\mathbf{H}_{3} e^{j\left(\delta_{21}-\delta_{31}\right)}\right] .
\end{array}\right.
$$

Note that the right hand sides of Eqs. (42) do not depend on $\theta_{1}$ but on $\delta_{21}$ and $\delta_{31}$.

Finally, combining Eqs. (42b) and (42d) as $\left(n_{1}(42 \mathrm{~d})-\right.$ $\left.n_{2}(42 \mathrm{~b})\right) / n_{1} n_{2}$ and Eqs. (42b) and (42f) as $\left(n_{1}(42 \mathrm{f})-\right.$ $\left.n_{3}(42 \mathrm{~b})\right) / n_{1} n_{3}$ and grouping with Eqs. (42a), (42c) and (42e), we obtained the close form differential equations

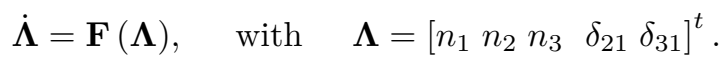

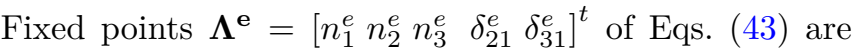
computed by solving $\mathbf{F}\left(\boldsymbol{\Lambda}^{\mathrm{e}}\right)=0$ and associated stability property are found by looking the sign of the real parts of the eigenvalues $\lambda_{i}$ (with $i \in[1,5]$ ) of the Jacobian matrix of $\mathbf{F}$ evaluated at $\boldsymbol{\Lambda}^{\mathbf{e}}$.

This analysis permits to predict the existence of stable periodic response regimes which correspond to the case where the real parts of all the eigenvalues are negative. In the following section, an asymptotic analysis of the complexified-averaged model is developed to characterize response regimes when stable property is not satisfied.

\subsection{Asymptotic analysis of the complexified- averaged model}

In this section we assume that $\epsilon \ll 1$ (i.e the mass of the NES is small with respect to the total mass of the fuselage and the blades) and that the parameters $\tilde{\lambda}_{y}, \tilde{\lambda}_{\delta}, \tilde{S}_{d}, \tilde{S}_{c}, \tilde{\mu}$ and $\tilde{\alpha}_{3}$ are of order $\epsilon$ (i.e $\tilde{\lambda}_{y}, \tilde{\lambda}_{\delta}, \tilde{S}_{d}, \tilde{S}_{c}, \tilde{\mu}, \tilde{\alpha}_{3} \sim O(\epsilon)$ ). These parameters are rescaling as

$$
\begin{aligned}
& \lambda_{y}=\frac{\tilde{\lambda}_{y}}{\epsilon} ; \quad S_{d}=\frac{\tilde{S}_{d}}{\epsilon} ; \quad \mu=\frac{\tilde{\mu}}{\epsilon} ; \\
& \lambda_{\delta}=\frac{\tilde{\lambda}_{\delta}}{\epsilon} ; \quad S_{c}=\frac{\tilde{S}_{c}}{\epsilon} ; \quad \alpha_{3}=\frac{\tilde{\alpha}_{3}}{\epsilon} .
\end{aligned}
$$

with $\lambda_{y}, \lambda_{\delta}, S_{d}, S_{c}, \mu, \alpha_{3} \sim O(1)$. 
Moreover, we focus the analysis for $\Omega$ around $\omega_{y}+\omega_{\delta}$ introducing the detuning term $a$, defined as

$$
\Omega=\omega_{y}+\omega_{\delta}+a \epsilon,
$$

with $a \sim O(1)$.

Using the rescaled parameters, the terms $\mathbf{R}_{31}, \mathbf{L}_{31}$ and $\alpha_{r, 1}$ can be expanded in a first-order Taylor series around $\epsilon=0$ giving

$$
\begin{aligned}
\mathbf{R}_{31} & =j+O\left(\epsilon^{2}\right) \\
\mathbf{L}_{31} & =-\frac{\lambda_{\delta}}{8 \omega_{\delta}} \epsilon+j\left(\frac{\omega_{y}+a \epsilon}{4 \omega_{\delta}}\right)+O\left(\epsilon^{2}\right) \\
\alpha_{r, 1} & =-\frac{\lambda_{\delta}}{2} \epsilon+j\left(\omega_{y}+a \epsilon\right)+O\left(\epsilon^{2}\right) .
\end{aligned}
$$

Introducing Eqs. (46), (47) and (48) (neglecting the $O\left(\epsilon^{2}\right)$ terms) and the rescaled parameters (44), Eqs. (39) becomes

$$
\left\{\begin{array}{c}
\dot{\phi}_{1}+\epsilon\left(\frac{\lambda_{y}+j \omega_{y}}{2} \phi_{1}-j \frac{\omega_{y}}{2} \phi_{2}-S_{d} \omega_{y} \phi_{3}\right)=0 \\
\dot{\phi}_{2}+\frac{\epsilon \lambda_{y}}{2} \phi_{1}-j \frac{\omega_{y}}{2}\left(\phi_{1}-\phi_{2}\right)(1-\epsilon)-\epsilon S_{d} \omega_{y} \phi_{3}+ \\
\phi_{2}(1+\epsilon)\left(\frac{\mu}{2}-j \frac{3 \alpha_{3}}{8 \omega_{y}^{3}}\left|\phi_{2}\right|^{2}\right)=0 \\
\dot{\phi}_{3}+\epsilon\left(\left(\frac{\lambda_{\delta}}{2}-j a\right) \phi_{3}-\frac{S_{c} \omega_{y}^{2}}{8 \omega_{\delta}} \phi_{1}\right)=0
\end{array}\right.
$$

Eqs. (49) define a simplified version of the complexifiedaveraged system which is called full slow-flow system and can be written as follow:

$$
\left\{\begin{array}{l}
\dot{\phi}_{2}=f_{2}\left(\phi_{1}, \phi_{2}, \phi_{3}, \epsilon\right) \\
\dot{\phi}_{1}=\epsilon f_{1}\left(\phi_{1}, \phi_{2}, \phi_{3}\right) \\
\dot{\phi}_{3}=\epsilon f_{3}\left(\phi_{1}, \phi_{2}, \phi_{3}\right),
\end{array}\right.
$$

which highlights the "slow/fast" nature of the system. Here we prefer to use the terminology introduced by Gendelman and Bar [15] for which the terms fast and slow are replaced by slow and super-slow respectively, whereby the term fast is reserved for the fast component $e^{j \omega_{y} t}$. Therefore, system (50) consists of one slow complex variable $\phi_{2}$ and two super-slow variables $\phi_{1}$ and $\phi_{3}$.

Eqs. (50) can be reformulated by switching from the slow time scale $t$ to the super-slow time scale $\tau=\epsilon t$ as

$$
\left\{\begin{array}{l}
\epsilon \Phi_{2}^{\prime}=f_{2}\left(\Phi_{1}, \Phi_{2}, \Phi_{3}, \epsilon\right) \\
\Phi_{1}^{\prime}=f_{1}\left(\Phi_{1}, \Phi_{2}, \Phi_{3}\right) \\
\Phi_{3}^{\prime}=f_{3}\left(\Phi_{1}, \Phi_{2}, \Phi_{3}\right),
\end{array}\right.
$$

where ${ }^{\prime}=\frac{d}{d \tau}$ and $\Phi_{i}(\tau)=\phi_{i}\left(t=\frac{\tau}{\epsilon}\right)$. Solutions of the super-slow/slow system (50) (or (51)) can exhibit slow and super-slow epochs characterized by the speed at which the solution advances.

Stating $\epsilon=0$, the following subsystems are derived from
(50) and (51) respectively:

$$
\left\{\begin{array}{l}
\dot{\phi}_{2}=f_{2}\left(\phi_{1}, \phi_{2}, \phi_{3}, 0\right) \\
\dot{\phi}_{1}=0 \\
\dot{\phi}_{3}=0
\end{array}\right.
$$

which are the slow subsystem, and

$$
\left\{\begin{array}{l}
0=f_{2}\left(\Phi_{1}, \Phi_{2}, \Phi_{3}, 0\right) \\
\Phi_{1}^{\prime}=f_{1}\left(\Phi_{1}, \Phi_{2}, \Phi_{3}\right) \\
\Phi_{3}^{\prime}=f_{3}\left(\Phi_{1}, \Phi_{2}, \Phi_{3}\right),
\end{array}\right.
$$

which are the super-slow subsystem.

In the following sections the geometric singular perturbation theory (GSPT) $[22,27,28]$ is used to describe the dynamics of the full system (50) (and (51)) for $0<\epsilon \ll 1$ from the analysis of the slow and super-slow subsystems (52) and (53) (which are defined for $\epsilon=0$ ). More precisely, we use the following result of the GSPT: if $0<\epsilon \ll 1$, the dynamics of the full system (50) (or (51)) during slow (resp. super-slow) epoch is given by the dynamic of the slow (resp. super-slow) subsystem (52) (resp. (53)).

\subsubsection{The Critical Manifold and its stability}

The algebraic equation (53a) of the super-slow subsystem defines the so-called Critical Manifold (CM) [27]

$$
C M:=\left\{\left(z_{1}, z_{2}, z_{3}\right) \in \mathbb{C}^{3} \mid f_{2}\left(z_{1}, z_{2}, z_{3}, 0\right)=0\right\} .
$$

Because they annul $\dot{\phi}_{2}$ in (52), the points of the CM are fixed points for the slow subsystem. From Eq. (53a) the $\mathrm{CM}$ can take the following form

$$
\Phi_{1}(\tau)=\Phi_{2}(\tau) F\left(\left|\Phi_{2}(\tau)\right|\right), \forall \Phi_{3},
$$

where the complex function $F$ is defined by

$$
F(x)=F_{R}(x)+j F_{I}(x)=1-\frac{3 \alpha_{3}}{4 \omega_{y}^{4}} x^{2}-j \frac{\mu}{\omega_{y}} .
$$

The form of the CM given by Eqs. (55-56) as been introduced by Starosvetsky and Gendelman $[10]^{6}$ to study harmonic forced linear system with NES and it is also found in studies of nonlinear self-excitated system with NES, see for example $[19,15]$. Previous cited references study system involving only the two variables $\phi_{1}$ and $\phi_{2}$ (there is a unique primary system coupled to a NES), therefore the $\mathrm{CM}$ is defined in $\mathbb{C}^{2}$. The distinctive feature of this work is the fact that the $\mathrm{CM}$ is defined in $\mathbb{C}^{3}$, keeping the same form in the $\left(\phi_{1}, \phi_{2}\right)$-space.

It is convenient to characterize the CM in $\mathbb{R}$. To achieve this, again polar coordinates are introduced

$$
\Phi_{i}(\tau)=N_{i}(\tau) e^{j \Theta_{j}(\tau)} \text { for } i=1,2 \text { and } 3,
$$

\footnotetext{
${ }^{6}$ In the literature devoted to the NES (for example in [10] and in many other sources) the Critical Manifold is called Slow Invariant Manifold (with the typical acronym SIM).
} 


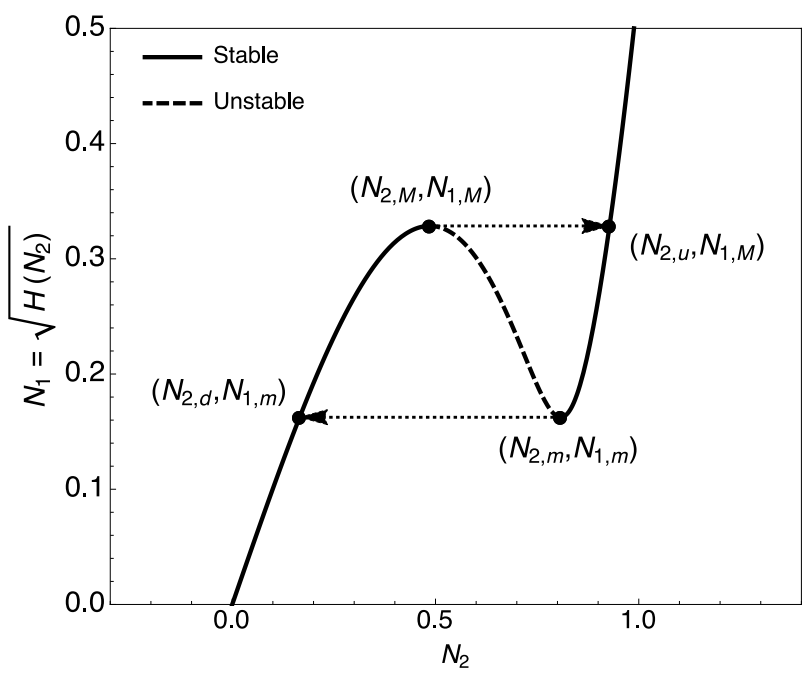

(a)

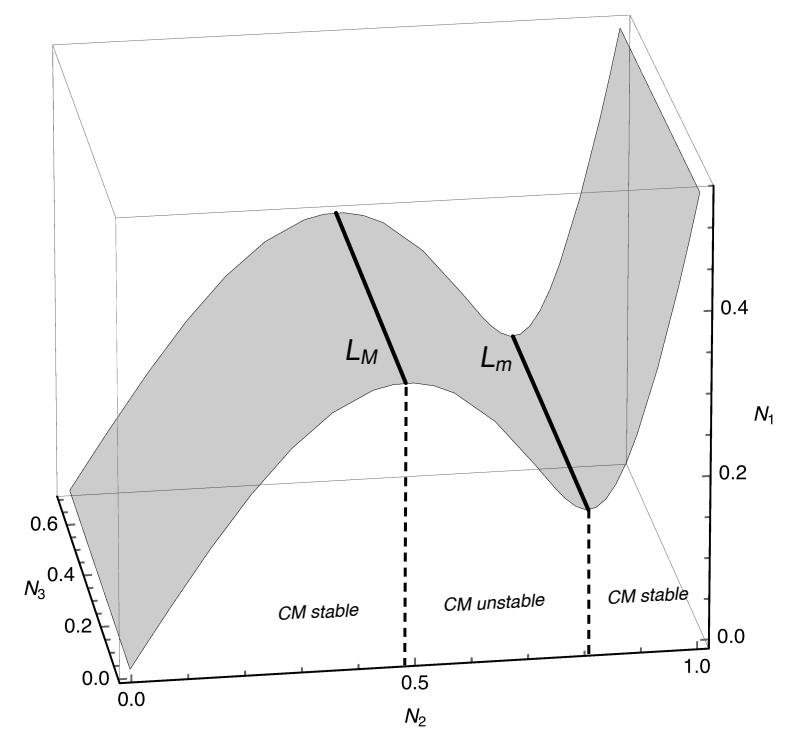

(b)

Figure 5: Critical Manifold (CM). Following parameters are used: $\omega_{y}=1, \alpha_{3}=2$ and $\mu=0.2$. (a) In the $\left(N_{1}, N_{2}\right)$-plan and (b) In the $\left(N_{1}, N_{2}, N_{3}\right)$-space.

and we compute successively the module and the argument of (55), that lies to

$$
\left\{\begin{array}{l}
N_{1}^{2}=N_{2}^{2}\left[F_{R}\left(N_{2}\right)^{2}+F_{I}\left(N_{2}\right)^{2}\right]=H\left(N_{2}\right), \forall N(58 \mathrm{a}) \\
\Theta_{1}=\Theta_{2}+\arctan \left(\frac{F_{I}\left(N_{2}\right)}{F_{R}\left(N_{2}\right)}\right), \forall \Theta_{3} .
\end{array}\right.
$$

The local extrema of the function $H(x)$ are given by the positive roots of its derivative $H^{\prime}(x)$. An easy calculus shows that the local extrema occur at

$$
\begin{aligned}
& N_{2, M}=\frac{2}{3} \omega_{y}^{2} \sqrt{\frac{2-\sqrt{1-3 \frac{\mu^{2}}{\omega_{y}^{2}}}}{\alpha_{3}}} \\
& N_{2, m}=\frac{2}{3} \omega_{y}^{2} \sqrt{\frac{2+\sqrt{1-3 \frac{\mu^{2}}{\omega_{y}^{2}}}}{\alpha_{3}}}
\end{aligned}
$$

if the following relation holds

$$
\mu<\frac{1}{\sqrt{3}} \omega_{y}
$$

and in this case $N_{2, M}<N_{2, m}$.

Stability of the Critical Manifold. To determine the stability of the CM, we must know if the CM attract or repel the slow dynamics. For that, using the polar coordinates $\phi_{i}(t)=n_{i}(t) e^{j \theta_{i}(t)}$, we rewrite the slow subsystem (52) as follow

$$
\left\{\begin{array}{l}
\frac{\partial n_{1}}{\partial t}=0 \\
\frac{\partial n_{2}}{\partial t}=\frac{\omega_{y}}{2}\left(n_{1} \sin \delta_{21}+n_{2} F_{I}\left(n_{2}\right)\right) \\
\frac{\partial n_{3}}{\partial t}=0 \\
\frac{\partial \delta_{21}}{\partial t}=\frac{\omega_{y}}{2}\left(\frac{n_{1}}{n_{2}} \cos \delta_{21}-F_{R}\left(n_{2}\right)\right) \\
\frac{\partial \delta_{31}}{\partial t}=0 .
\end{array}\right.
$$

where (as in Sect. 4.2) the argument difference $\delta_{21}=\theta_{2}-\theta_{1}$ and $\delta_{31}=\theta_{3}-\theta_{1}$ have been introduced. Stability range of the $\mathrm{CM}$ is then determined by examining the sign of the eigenvalues real parts of the Jacobian matrix of differential system (62b-62d) on the CM. It can be shown that the condition of stability of the $\mathrm{CM}$ is equivalent to

$$
H^{\prime}\left(N_{2}\right)>0
$$

and the stability range of the $\mathrm{CM}$ is characterized by the points $\left(N_{2, m}, N_{1, m}\right)$ and $\left(N_{2, M}, N_{1, M}\right)$ where $N_{1, M}=$ $\sqrt{H\left(N_{2, M}\right)}, \quad N_{1, m}=\sqrt{H\left(N_{2, m}\right)}$, which are therefore called fold points [29]. A typical Critical Manifold and it stability range are depicted (see Fig. $5(\mathrm{a}))$ in $\left(N_{2}, N_{1}\right)$-plan where $N_{2, d}$ and $N_{2, u}$ are solutions of

$H\left(N_{2, m}\right)=H\left(N_{2, d}\right) \Rightarrow N_{2, d}=\frac{2 \sqrt{2}}{3} \omega_{y}^{2} \sqrt{\frac{1-\sqrt{1-3 \frac{\mu^{2}}{\omega_{y}^{2}}}}{\alpha_{3}}}$, 


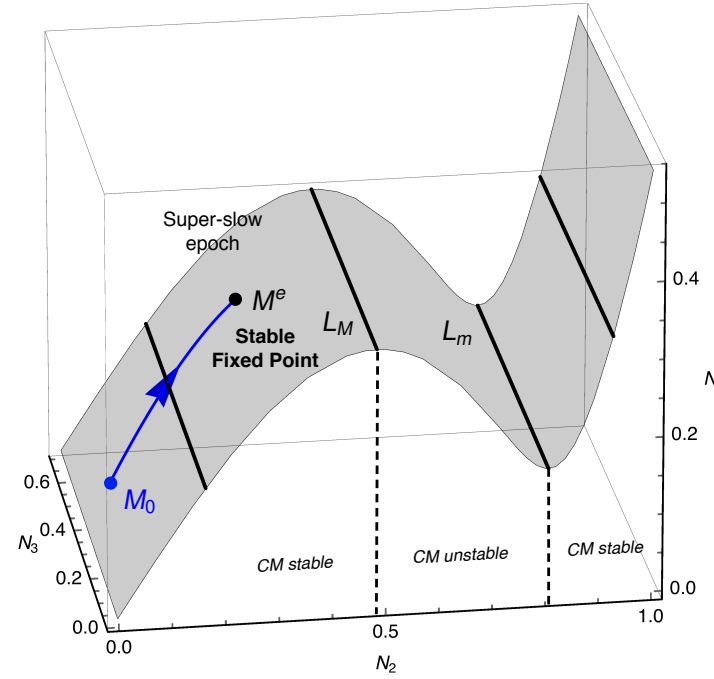

(a)

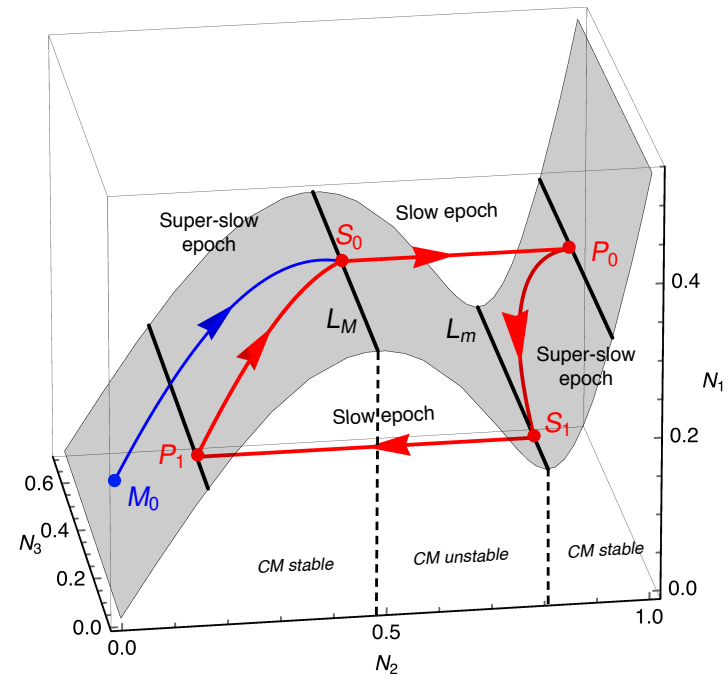

(c)

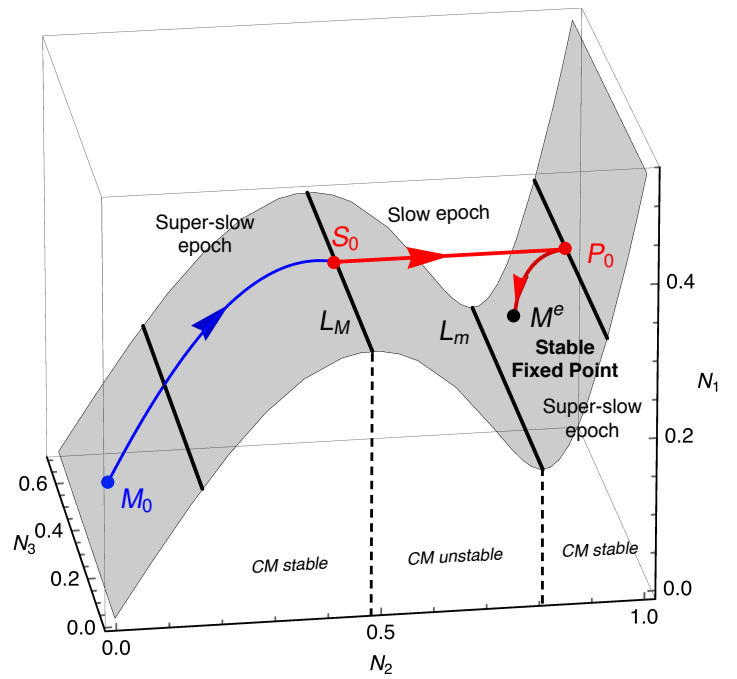

(b)

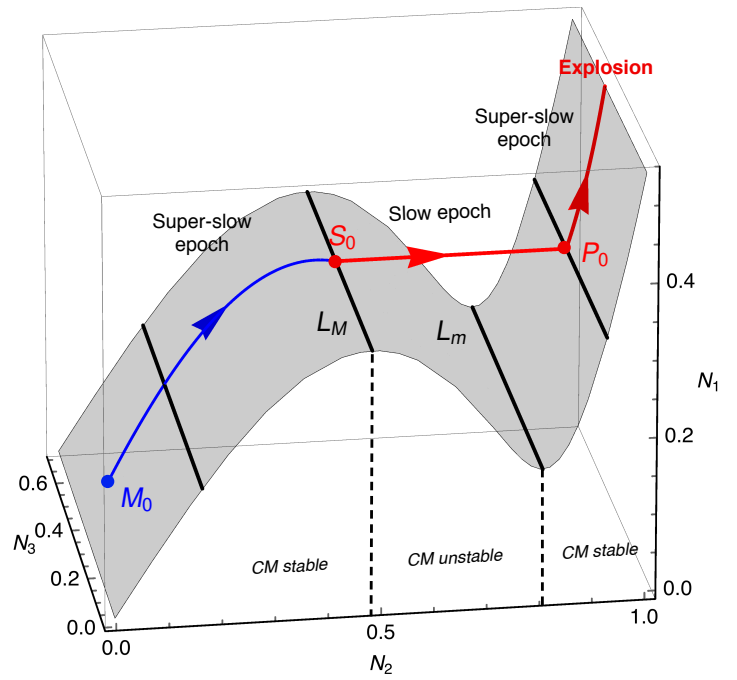

(d)

Figure 6: Outline schematic showing the possible steady-state regimes of the full slow-flow system (49): (a) a stable fixed point

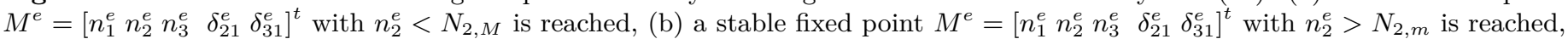
(c) relaxation oscillations and (d) explosion.

and

$H\left(N_{2, M}\right)=H\left(N_{2, u}\right) \Rightarrow N_{2, u}=\frac{2 \sqrt{2}}{3} \omega_{y}^{2} \sqrt{\frac{1+\sqrt{1-3 \frac{\mu^{2}}{\omega_{y}^{2}}}}{\alpha_{3}}}$,

respectively. In the $\left(N_{1}, N_{2}, N_{3}\right)$-space, each fold point defines a folded line $\left(L_{M}\right.$ and $\left.L_{m}\right)$ co-linear to the $N_{3}$-axis (see Fig. 5(b)).

The shape and the stability property of the CM (i.e. the existence of folded lines on which the stability of the CM changes) shown in Fig. 5 allow to define three steady-state regimes of the full slow-flow system (49) that can explain the three steady-state regimes of the SHM+NES observed in Figs. 4(b)-4(d).

To describe the steady regimes of the slow flow we consider situations on which, after an transient regime, the trajectory of the system arrives at a point $M_{0}$ on the CM. After that, three steady-state regimes may be considered (see Fig. 6):

A fixed point of the slow-flow is reached. In this case two scenarios must be considered which are depicted in Figs. 6(a) and 6(b). In the first scenario, a super-slow evolution, described by Eqs. (53), brings the slow-flow system

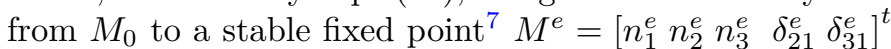
and the folded line $L_{M}$ is not reached (i.e. $n_{2}^{e}<N_{2, M}$, see Fig. 6(a)). In the second scenario, the folded line $L_{M}$ is reached at $S_{0}$ and the system jumps to $P_{0}$ (the jump corresponds to a slow epoch described by Eqs. (52)), which is followed by a super-slow evolution (i.e. a new super-

\footnotetext{
${ }^{7}$ As in Sect. 4.2, fixed point of the slow-flow must be understand as the fixed points of the real system deduced from full slow flow system (49) using polar coordinates $\phi_{i}(t)=n_{i}(t) e^{j \theta_{i}(t)}$ and considering argument differences $\delta_{i 1}=\theta_{i}(t)-\theta_{1}(t)$.
} 
slow epoch described again by Eqs. (53)) of the trajectory of the system, in the stable domain of the CM, until it reaches a stable fixed point $M^{e}$ characterized by $n_{2}^{e}>N_{2, m}$. These situations corresponds to a periodic solution of the SHM+NES (see Sect. 4.2 and Fig. 4(b)).

Relaxation oscillations. As for the second scenario described above, a super-slow evolution brings the slow-flow system from $M_{0}$ to $S_{0} \in L_{M}$ (see Fig. 6(c)) but after $P_{0}$ a scenario of relaxation oscillations [30] is observed: after the first jump the super-slow evolution brings the system from $P_{0}$ to $S_{1} \in L_{m}$. After another jump from $S_{1}$ to $P_{1}$ and a super-slow evolution, the trajectory returns to $S_{0}$ and so on (see Fig. 5(b)). Such scenario of relaxation oscillations for the slow-flow system can explain the existence of Strongly Modulated Responses [19, 15, 10] for the SHM+NES (as observed Fig. 4(c)).

Explosion. Until $P_{1}$ the slow-flow evolves the same way as for relaxation oscillations mechanism. However, instead of moving toward a stable fixed point or the folded line $L_{m}$, the trajectory of the system follows the CM to the infinity (see Fig. 6(d)). This scenario explains no suppression regime for the SHM+NES (as observed Fig. 4(d)).

The existence of one of the three steady-state regimes described above or an other depends of the position and the stability of the fixed points of the full slow-flow system (49). Indeed, a stable fixed point of the full slow flow placed on the stable part of the CM is a necessary condition to obtain PRs of the SHM+NES (31). On the other hand, the relaxation oscillations of the slow flow (or SRMs for the SHM+NES) can exist if both folded lines $L_{M}$ and $L_{m}$ have attractive parts. Position and stability of the fixed points of the full slow-flow system and attractivity (or repulsively) of the folded lines are determined in the next section through the study of the super-slow subsystem (53).

\subsubsection{Fixed points (and their stability) and folded singu-} larities of the slow-flow

Introducing the CM Eq. (55) in Eqs. (53b) and (53c), the super-slow subsystem is written only with respect to the variables $\Phi_{2}$ and $\Phi_{3}$

$$
\left\{\begin{array}{c}
\frac{\partial\left[\Phi_{2} F\left(\left|\Phi_{2}\right|\right)\right]}{\partial \tau}=-\frac{\lambda_{y}}{2} \Phi_{2} F\left(\left|\Phi_{2}\right|\right)- \\
j \frac{\omega_{y}}{2} \Phi_{2}\left(F\left(\left|\Phi_{2}\right|\right)-1\right)+S_{d} \omega_{y} \Phi_{3} \\
\frac{\partial \Phi_{3}}{\partial \tau}=-\left(\frac{\lambda_{\delta}}{2}-j a\right) \Phi_{3}+\frac{S_{c} \omega_{y}^{2}}{8 \omega_{\delta}} \Phi_{2} F\left(\left|\Phi_{2}\right|\right)
\end{array}\right.
$$

Using the polar coordinates (57) and separating real and imaginary parts, Eqs. (66) takes the following form

$$
\left\{\begin{array}{l}
F_{R} \frac{\partial N_{2}}{\partial \tau}-N_{2} \frac{\partial \Theta_{2}}{\partial \tau} F_{I}+N_{2} \frac{\partial N_{2}}{\partial \tau} F_{R}^{\prime}= \\
-\frac{\lambda}{2} N_{2} F_{R}+\frac{\omega_{y}}{2} N_{2} F_{I}+S_{d} \omega_{y} N_{3} \cos \left(\Delta_{32}\right) \quad(67 \mathrm{a}) \\
N_{2} \frac{\partial \Theta_{2}}{\partial \tau} F_{R}+\frac{\partial N_{2}}{\partial \tau} F_{I}+N_{2} \frac{\partial N_{2}}{\partial \tau} F_{I}^{\prime}= \\
-\frac{\lambda}{2} N_{2} F_{I}-\frac{\omega_{y}}{2} N_{2}\left(F_{R}-1\right)+S_{d} \omega_{y} N_{3} \sin \left(\Delta_{32}(67 \mathrm{~b})\right. \\
\frac{\partial N_{3}}{\partial \tau}=-\frac{\lambda}{2} N_{3}+ \\
\frac{S_{c} \omega_{y}^{2}}{8 \omega_{\delta}} N_{2}\left(F_{R} \cos \left(\Delta_{32}\right)+F_{I} \sin \left(\Delta_{32}\right)\right) \\
\frac{\partial \Theta_{3}}{\partial \tau}=a N_{3}+ \\
\frac{S_{c} \omega_{y}^{2}}{8 \omega_{\delta}} N_{2}\left(F_{I} \cos \left(\Delta_{32}\right)-F_{R} \sin \left(\Delta_{32}\right)\right),
\end{array}\right.
$$

involving the argument difference $\Delta_{32}=\Theta_{3}-\Theta_{2}$. Combining Eqs (67a-67d), system of Eqs. (67) can be finally reduced (after some calculation steps) to the following form

$$
\left\{\begin{array}{l}
g\left(N_{2}\right) \frac{\partial N_{2}}{\partial \tau}=f_{N_{2}}\left(N_{2}, N_{3}, \Delta_{32}\right) \\
g\left(N_{2}\right) \frac{\partial \Delta_{32}}{\partial \tau}=f_{\Delta_{32}}\left(N_{2}, N_{3}, \Delta_{32}\right) \\
\frac{\partial N_{3}}{\partial \tau}=f_{N_{3}}\left(N_{2}, N_{3}, \Delta_{32}\right)
\end{array}\right.
$$

where

$$
g(x)=\frac{H^{\prime}(x)}{2}
$$

$$
\begin{aligned}
& f_{N_{2}}\left(N_{2}, N_{3}, \Delta_{32}\right)=a_{11} \cos \Delta_{32}+a_{12} \sin \Delta_{32}-c_{1}, \\
& f_{\Delta_{32}}\left(N_{2}, N_{3}, \Delta_{32}\right)=a_{21} \cos \Delta_{32}+a_{22} \sin \Delta_{32}-c_{2}, \\
& f_{N_{3}}\left(N_{2}, N_{3}, \Delta_{32}\right)=a_{31} \cos \Delta_{32}+a_{32} \sin \Delta_{32}-c_{3}, \\
& a_{11}=N_{2} N_{3} S_{d} \omega_{y} F_{R}, \\
& a_{12}=N_{2} N_{3} S_{d} \omega_{y} F_{I}, \\
& a_{21}=g\left(N_{2}\right) \frac{N_{2} S_{c} \omega_{y}^{2}}{N_{3} 8 \omega_{\delta}} F_{I}+N_{3} S_{d} \omega_{y}\left(F_{I}+F_{I}^{\prime} N_{2}\right), \\
& a_{21}=-g\left(N_{2}\right) \frac{N_{2} S_{c} \omega_{y}^{2}}{N_{3} 8 \omega_{\delta}} F_{R}+N_{3} S_{d} \omega_{y}\left(F_{R}+F_{R}^{\prime} N_{2}\right), \\
& a_{31}=\frac{N_{2} S_{c} \omega_{y}^{2}}{8 \omega_{\delta}} F_{R}, \\
& a_{32}=\frac{N_{2} S_{c} \omega_{y}^{2}}{8 \omega_{\delta}} F_{I},
\end{aligned}
$$

and,

$$
\begin{aligned}
c_{1} & =\frac{N_{2}^{2}}{2}\left(\lambda_{y}\left(F_{R}^{2}+F_{I}^{2}\right)-F_{I} \omega_{y}\right), \\
c_{2} & =-N_{2} \frac{\omega_{y}}{2}\left(F_{I}^{2}+F_{I} F_{I}^{\prime} N_{2}+\left(F_{R}-1\right)\left(F_{R}+F_{R}^{\prime} N_{2}\right)\right) \\
& -\lambda_{y} N_{2}^{2}\left(F_{I} F_{R}^{\prime}-F_{I}^{\prime} F_{R}\right)+2 a g\left(N_{2}\right), \\
c_{3} & =N_{3} \frac{\lambda_{\delta}}{2} .
\end{aligned}
$$

From Eqs. (68), it is possible to detect fixed points and folded singularities.

Fixed points. The (regular) fixed points of Eqs. (68), $\left\{N_{2}^{e}, N_{3}^{e}, \Delta_{32}^{e}\right\}$, are defined by: 


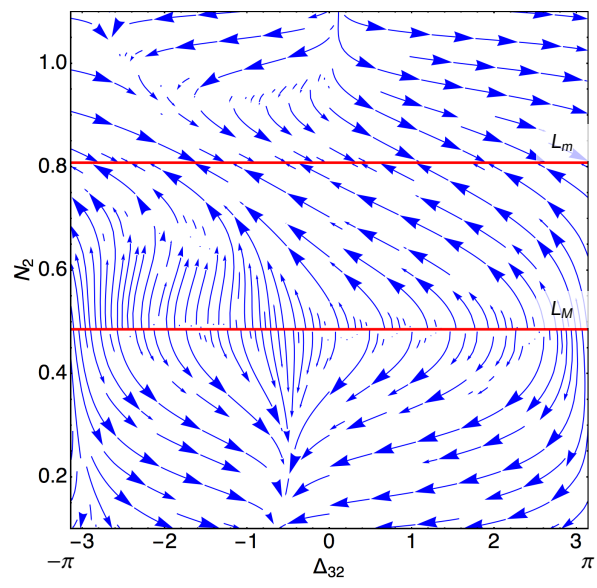

(a)

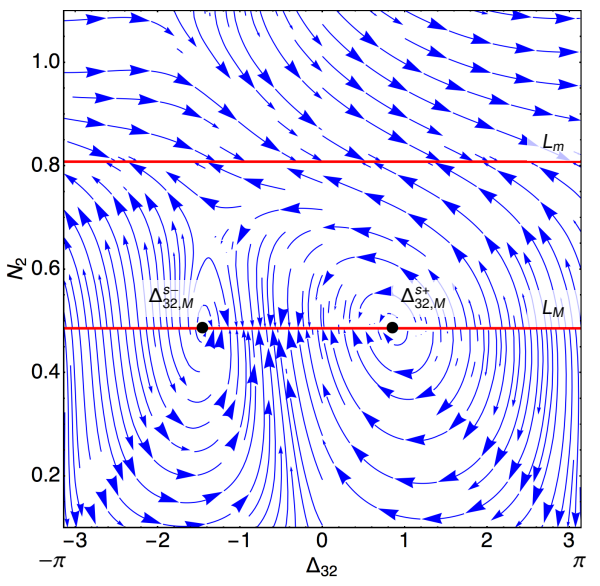

(b)

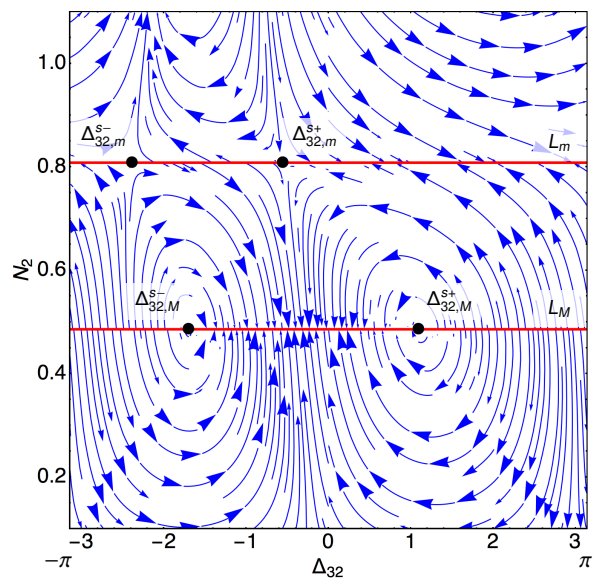

(c)

Figure 7: Phase portraits of the desingularized super-slow subsystem (76) for constant value of $N_{3}$. To take into account that the desingularization reverses the direction of time on the repelling part of the CM, the direction of the flow is reversed (i.e. the arrows are reversed) between $L_{M}$ and $L_{m}$. Parameters used: $\omega_{y}=1, \alpha_{3}=2, \mu=0.2, S_{c}=2, S_{d}=1, \lambda_{y}=0.3, \lambda_{\delta}=0.2$ and $a=-0.4$. For this set of parameters we have: $N_{3, M}^{c r}=0.121$ and $N_{3, m}^{c r}=0.426$. (a) $N_{3}=0.02<N_{3, M}^{c r}$, (b) $N_{3, M}^{c r}<N_{3}=0.3<N_{3, m}^{c r}$ and (c) $N_{3}=0.7>N_{3, m}^{c r}$.

$$
\begin{aligned}
f_{N_{2}}\left(N_{2}^{e}, N_{3}^{e}, \Delta_{32}^{e}\right) & =0 \\
f_{\Delta_{32}}\left(N_{2}^{e}, N_{3}^{e}, \Delta_{32}^{e}\right) & =0 \\
f_{N_{3}}\left(N_{2}^{e}, N_{3}^{e}, \Delta_{32}^{e}\right) & =0 \\
g\left(N_{2}^{e}\right) & \neq 0 .
\end{aligned}
$$

If $\epsilon \ll 1$, fixed points computed from Eqs. (75) corresponds to fixed points of the system (43) obtained in Sect. 4.2. As usual, stability of the fixed points are found by looking the sign of the eigenvalues real parts of the Jacobian matrix of the vector function $\mathbf{F}_{\mathbf{1}}=\left(f_{N_{2}} / g, f_{\Delta_{32}} / g, f_{N_{3}}\right)$ evaluated at $\left\{N_{2}^{e}, N_{3}^{e}, \Delta_{32}^{e}\right\}$.

Folded singularities. The folded singularities $\left\{N_{2, m / M}, N_{3}^{s}, \Delta_{32}^{s}\right\}$ (where $N_{2, m / M}$ denotes indistinctly $N_{2, M}$ or $N_{2, m}$ ) are points of the folded lines $L_{M}$ and $L_{m}$ at which the attractivity of the folded lines changes. Folded singularities are defined as the fixed points of the desingularized super-slow subsystem, which is obtained introducing the time rescaling $\tau \rightarrow \tau^{\prime} g\left(N_{2}\right)$ [28]

$$
\left\{\begin{array}{l}
\frac{\partial N_{2}}{\partial \tau^{\prime}}=f_{N_{2}}\left(N_{2}, N_{3}, \Delta_{32}\right) \\
\frac{\partial \Delta_{32}}{\partial \tau^{\prime}}=f_{\Delta_{32}}\left(N_{2}, N_{3}, \Delta_{32}\right) \\
\frac{\partial N_{3}}{\partial \tau^{\prime}}=g\left(N_{2}\right) f_{N_{3}}\left(N_{2}, N_{3}, \Delta_{32}\right) .
\end{array}\right.
$$

Note that because $g\left(N_{2}\right)<0$ between the $N_{2, M}$ and $N_{2, m}$, the previous time rescaling reverses the direction of time on the repelling part of the CM. Since $g\left(N_{2, m / M}\right)=0$, folded singularities are defined from (76) by

$$
\begin{aligned}
f_{N_{2}}\left(N_{2, m / M}, N_{3}^{s}, \Delta_{32}^{s}\right) & =0, \\
f_{\Delta_{32}}\left(N_{2, m / M}, N_{3}^{s}, \Delta_{32}^{s}\right) & =0 .
\end{aligned}
$$

The folded singularities are obtained first solving the (linear) system of Eqs. (77) with respect to $\left(\cos \Delta_{32}^{s}, \sin \Delta_{32}^{s}\right)$ (see Eqs. (70) and (71)). The associated determinant of this linear system satisfies:

$$
a_{11} a_{22}-a_{21} a_{12}=-S_{d}^{2} \omega_{y}^{2} N_{3}^{2} g\left(N_{2, m / M}\right)=0
$$

showing that one of the two equations (77a) and (77b) can be removed. Therefore, the folded singularities can be only defined by

$$
f_{N_{2}}\left(N_{2, m / M}, N_{3}^{s}, \Delta_{32}^{s}\right)=0 .
$$

Eq. (79) reads as (using Eqs. (70) and (73))

$$
\begin{array}{r}
a_{11}\left(N_{2, m / M}, N_{3}^{s}\right) \cos \Delta_{32}^{s}+a_{12}\left(N_{2, m / M}, N_{3}^{s}\right) \sin \Delta_{32}^{s}= \\
c_{1}\left(N_{2, m / M}, N_{3}^{s}\right), \quad(80)
\end{array}
$$

which can be solved with respect to $\Delta_{32}^{s}$ giving the following four solutions (2 for each folded line)

$$
\begin{aligned}
& \Delta_{32, m / M}^{s \pm}=\arcsin \left(\frac{a_{12}\left(N_{2, m / M}, N_{3}^{s}\right)}{\sqrt{a_{11}\left(N_{2, m / M}, N_{3}^{s}\right)^{2}+a_{12}\left(N_{2, m / M}, N_{3}^{s}\right)^{2}}}\right) \\
\pm & \arccos \left(\frac{c_{1}\left(N_{2, m / M}, N_{3}^{s}\right)}{\sqrt{a_{11}\left(N_{2, m / M}, N_{3}^{s}\right)^{2}+a_{12}\left(N_{2, m / M}, N_{3}^{s}\right)^{2}}}\right),
\end{aligned}
$$


if the conditions

$$
\left|\frac{c_{1}\left(N_{2, m / M}, N_{3}^{s}\right)}{\sqrt{a_{11}\left(N_{2, m / M}, N_{3}^{s}\right)^{2}+a_{12}\left(N_{2, m / M}, N_{3}^{s}\right)^{2}}}\right| \leq 1,
$$

are satisfied.

Conditions (82) show that the folded singularities exist for values of $N_{3}^{s}$ defined through Eqs. (73) and (74) by the inequalities

$$
N_{3}^{s} \geq N_{3, m / M}^{c r}
$$

with the lower bounds, named critical values, are given by

$$
\begin{aligned}
& \quad N_{3, m / M}^{c r}= \\
& \frac{N_{2, m / M}\left(\lambda_{y}\left(F_{R}\left(N_{2, m / M}\right)^{2}+F_{I}\left(N_{2, m / M}\right)^{2}\right)-\omega_{y} F_{I}\left(N_{2, m / M}\right) b\right.}{2 S_{d} \sqrt{F_{R}\left(N_{2, m / M}\right)^{2}+F_{I}\left(N_{2, m / M}\right)^{2}}} .
\end{aligned}
$$

\section{Prediction of the steady-state response regimes of the SHM+NES}

The prediction of the steady-state response regimes of the SHM+NES (31) is obtained checking first the local stability property of the trivial equilibrium point of Eqs. (31) (see Sect. 3.1) and using the asymptotic analysis of the full slowflow system (49) to characterize the response regimes when the trivial equilibrium point of Eqs. (31) is unstable. From the asymptotic analysis of slow-flow system (49) performed in Sect. 4.3, we characterize

- the CM (55) and its the fold points $N_{2, M}$ and $N_{2, m}$ (see Eqs. (59) and (60)) and the points $N_{2, d}$ and $N_{2, u}$ defined in Eqs (64) and (65) respectively,

the stable periodic regimes of Eqs. (31) as the stable nontrivial fixed points of Eqs. (68),

- the non periodic response regimes (SMRs or no sup(84) pression) of Eqs. (31) from the unstable nontrivial fixed points of Eqs. (68).

Phase portraits of the desingularized super-slow subsystem (76) are plotted in Fig. 7. Note that, to take into account that the desingularization reverses the direction of time on the repelling part of the $\mathrm{CM}$, the direction of the flow is reversed (i.e. the arrows are reversed) between $L_{M}$ and $L_{m}$. A complete phase portrait must take into account the whole dynamics, including the variable $N_{3}$. However, to facilitate the interpretation of the phase portraits and understand the role of the folded singularities, the phase portraits are computed for constant values of $N_{3}$ and plotted in the plan $\left(\Delta_{32}, N_{2}\right)$. Three representative values of $N_{3}$ are chosen: $N_{3}<N_{3, M}^{c r}, N_{3, M}^{c r}<N_{3}<N_{3, m}^{c r}$ and $N_{3}>N_{3, m}^{c r}$. If $N_{3}<N_{3, M}^{c r}$ (see Fig. 7(a)), there are no folded singularities, and we notice that the folded line $L_{M}$ is repulsive and the folded line $L_{m}$ is attractive. The repulsivity of $L_{M}$ prevents jumps from $S_{0}$ to $P_{0}$ (see Fig. 6) and therefore sustained relaxation oscillations and explosion are not possible. If $N_{3, M}^{c r}<N_{3}<N_{3, m}^{c r}$ (see Fig. 7(b)), there are folded singularities $\Delta_{32, M}^{s \pm}$ on the folded line $L_{M}$ which becomes attractive between them. Since the attractivity of $L_{m}$ in unchanged, this situation allows the existence of sustained relaxation oscillations or explosion. Finally, if $N_{3}>N_{3, m}^{c r}$ (see Fig. $7(\mathrm{c})$ ), there are also folded singularities $\Delta_{32, m}^{s \pm}$ on the folded line $L_{m}$ which becomes repulsive between them. As long as $L_{m}$ have attractive part, this situation allows also the existence of sustained relaxation oscillations or explosion.

Finally, inequalities (83) can be therefore interpreted as a necessary condition to relaxation oscillations occur. Relaxation oscillations can be possible if during transient regime $N_{3}(t)$ reaches at least $N_{3, m}^{c r}$, allowing $N_{2}(t)$ to reach the folded line $L_{M}$ between $\Delta_{32, M}^{s+}$ and $\Delta_{32, M}^{s-}$ obtained from Eq. (81) evaluated with $N_{3}^{s}=N_{3}(t)$. Unfortunately, this scenario cannot be predicted a priori. From these results, it seems to be difficult for example to develop a tool to predict stability of relaxation oscillations as done in [10] with the $1 D$ mapping tool. In the sequel we will only verify if the senario is satisfied when the response regimes of Eqs. (34) is not periodic.
The study is restricted to the prediction of steady-state response regimes resulting from initial conditions not too far from the trivial equilibrium position of Eqs (34). Moreover, in the remaining of the section, analytical and numerical results are computed using the following set of parameters

$$
\begin{array}{ccc}
\omega_{y}=1, & \omega_{\delta}=2, \quad \lambda_{y}=0.3, \quad S_{c}=2, \\
S_{d}=1, \quad \alpha_{3}=2, \quad \mu=0.2, \quad \epsilon=0.01 .
\end{array}
$$

For this set of parameters the characteristic points of the CM are: $N_{2, M}=0.486, N_{2, m}=0.808, N_{2, u}=0.928$, $N_{2, u}=0.166$, and the critical values with respect to $N_{3}$ are: $N_{3, M}^{c r}=0.121$ and $N_{3, m}^{c r}=0.426$. Moreover, Eqs. (68) admits 0,1 or 2 fixed points.

The nature of the steady-response regimes is studied with respect to the damping coefficient of a blade $\lambda_{\delta}$ and the detuning parameter $a$ (defined in Eq. (45)).

The steady-state response regimes are classified in five domain:

\section{Domain 0 Complete suppression}

Domain 1 Partial suppression through PR

Domain 2 Partial suppression through PR or SMR

Domain 3 Partial suppression through SMR

Domain 4 No suppresion

The diagram of the Fig. 9 summarizes the algorithm used to determine the response regimes domains. Results are plotted in Fig. 10 in which each domain is represented by an area of the plane $\left(\lambda_{\delta}, a\right)$. Finally, for each domain (expect for Domain 0 which characterizes the Complete suppression) one or two examples are selected and studied deeply. Results are presented in Figs. 11 to 17. The values of $\lambda_{\delta}$ and $a$ used for these examples and the corresponded coordinates $N_{1}^{e}, N_{2}^{e}$ and $N_{3}^{e}$ of the fixed points of (68) are indicated in Tab. 1. 
Table 1: Values of $\lambda_{\delta}$ and $a$ used in Examples 1, 2a, 2b, 3a, 3b, 4a and 4b. Coordinates $N_{1}^{e}, N_{2}^{e}$ and $N_{3}^{e}$ of the corresponding fixed points of (68) are also indicated. $\mathrm{S} \equiv$ stable and $\mathrm{U} \equiv$ unstable.

\begin{tabular}{c|cc|c|cc}
\hline Example & $\boldsymbol{\lambda}_{\boldsymbol{\delta}}$ & $\boldsymbol{a}$ & $\begin{array}{c}\text { Number of } \\
\text { Fixed Pt. }\end{array}$ & Fixed Pt. 1: $\left\{N_{1}^{e}, N_{2}^{e}, N_{3}^{e}\right\}$ & Fixed Pt. 2: $\left\{N_{1}^{e}, N_{2}^{e}, N_{3}^{e}\right\}$ \\
\hline 1 & 0.35 & -0.4 & 2 & $\{0.323,0.433,0.157\} \mathrm{S}$ & $\{1.18,1.15,0.494\} \mathrm{U}$ \\
\hline 2 & 0.08 & 0 & 2 & $\{0.187,0.853,0.494\} \mathrm{S}$ & $\{1.19,1.16,1.04\} \mathrm{U}$ \\
\hline 2b & 0.06 & 0.5 & 1 & $\{0.203,0.865,0.581\} \mathrm{S}$ & - \\
\hline $3 \mathrm{a}$ & 0.2 & 0.4 & 1 & $\{0.223,0.712,0.269\} \mathrm{U}$ & - \\
\hline $3 \mathrm{~b}$ & 0.2 & -0.4 & 2 & $\{0.316,0.564,0.242\} \mathrm{U}$ & $\{0.853,1.08,0.532\} \mathrm{U}$ \\
\hline $4 \mathrm{a}$ & 0.1 & -0.7 & 2 & $\{0.293,0.614,0.356\} \mathrm{U}$ & $\{0.181,0.847,0.438\} \mathrm{U}$ \\
\hline $4 \mathrm{~b}$ & 0.035 & -0.4 & 0 & - & -
\end{tabular}

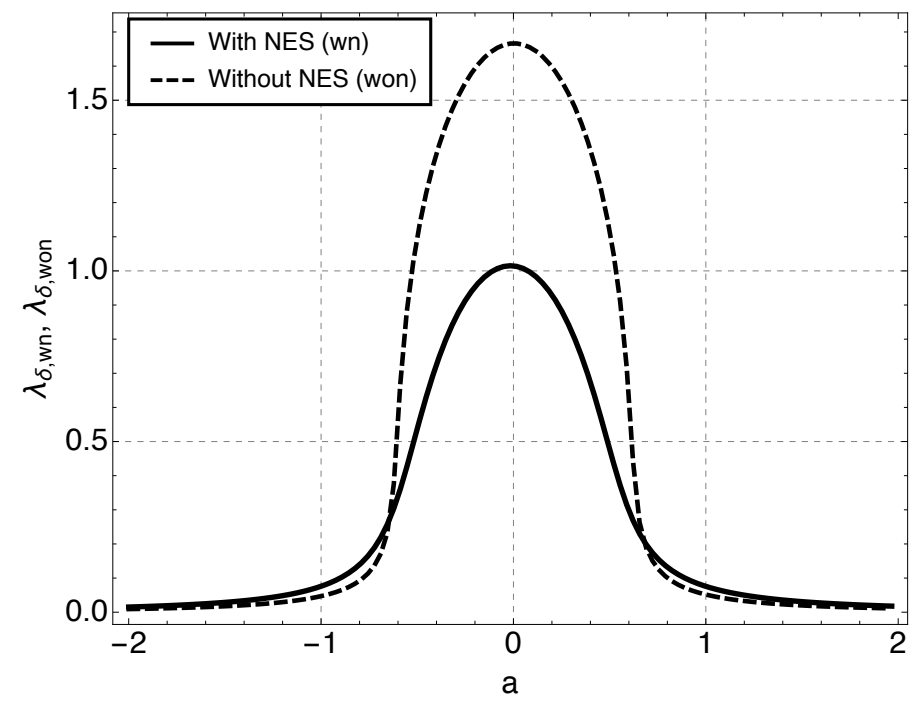

Figure 8: $\lambda_{\delta, \text { wn }}$ and $\lambda_{\delta \text {,won }}$ as a function of $a$. Parameters used: see Eq. (85).

\subsection{Domain 0: Complete suppression}

Analytical prediction of the complete suppression is performed in Sect. 3.1. However, it is interesting to compare the region of linear stability of the system with and without NES. To achieve that, the values of $\lambda_{\delta}$ that annul one of the eigenvalues of Eqs. (30) and Eqs. (31), denoted respectively $\lambda_{\delta \text {,won }}$ and $\lambda_{\delta \text {,wn }}$, are computed with respect to the parameter $a$ and plotted in Fig. 8. For each curve $\lambda_{\delta \text {,won }}(a)$ and $\lambda_{\delta \text {,wn }}(a)$, the area upper the curve corresponds to stable trivial solution and in the area under the curve the trivial solution in unstable. One can notice that for approximatively $-0.65 \lesssim a \lesssim 0.65$, the NES attachment favors the linear stability of the trivial solution. This is the opposite for $a \lesssim-0.65$ and $a \gtrsim 0.65$ but to a lesser extent.

The area upper the curve $\lambda_{\delta \text {,wn }}(a)$ defined the Domain 0 , it is depicted in gray in Fig. 10.

\subsection{Local stability of one of the fixed point of the slow-flow system: Domains 1 and 2}

Fixed points of the slow-flow system correspond to periodic solutions of the SHM+NES. Therefore, the domain of existence the Partial suppression through Periodic Response may correspond to the domain of local stability of one of the fixed point. However, as in Sect. 4.3.1 ( $\$$ A fixed point of the slow-flow is reached), the two following situations must be considered: $N_{2}^{e}<N_{2, M}$ and $N_{2}^{e}>N_{2, m}$ where $N_{2}^{e}$ is the $N_{2}$-coordinate of a stable fixed point. The two situations correspond to domain 1 and 2 respectively.

\subsubsection{Domain 1: partial suppression through PR}

This domain is represented by gray dots ("•") in Fig. 10. For $N_{2}^{e}<N_{2, M}$, the stable fixed point is reached before the folded line $L_{M}$. This corresponds to the scenario depicted in Fig. 6(a).

In this situation, relaxation oscillations or explosion of the slow-flow system and therefore SMR or no suppression regimes for the SHM+NES are avoided. Therefore, domain 1 corresponds to Partial suppression through Periodic Response. Fig. 11 shows an example of this situation.

\subsubsection{Domain 2: partial suppression through PR or $S M R$}

This domain is represented by black crosses (" $\times$ ") in Fig. 10. The case of one stable fixed point which satisfies the condition $N_{2}^{e}>N_{2, m}$ highlights the limit of the local stability study. Indeed, in this case, a least one jump from $N_{2, M}$ to $N_{2, u}$ is needed to reach the fixed point. After that, the fixed point can be really reached (as depicted in Fig. 6(b)) or sustained relaxation oscillations of the the slow-flow system are observed (as depicted in Fig. 6(c)). Examples of the two possible situations are shown in Figs. 12 (PR, Example 2a) and 13 (SMR, Example 2b).

To better understand the behavior of the system in these two situations, phase portraits of the desingularized superslow subsystem (76) is compared to the trajectory of the full slow-flow system (49) in the $\left(\Delta_{32}, N_{2}, N_{3}\right)$-space in Figs. 12(c) and 12(d) for example 2a and in Figs. 13(c) and $13(\mathrm{~d})$ for example $2 \mathrm{~b}$. For both examples $2 \mathrm{a}$ and $2 \mathrm{~b}$ a top view and an "optimized" view are presented. Only the end of the trajectory of the full slow-flow system (49) is plotted (see details in figures captions).

In Fig. 12(d) we can see that during its transient regimes the full slow-flow system reaches streamlines which bring it to the stable fixed point (green disk marker in Figs 12(d) and fig:Example1bc). On the contrary, in Fig. 13(d), we can 


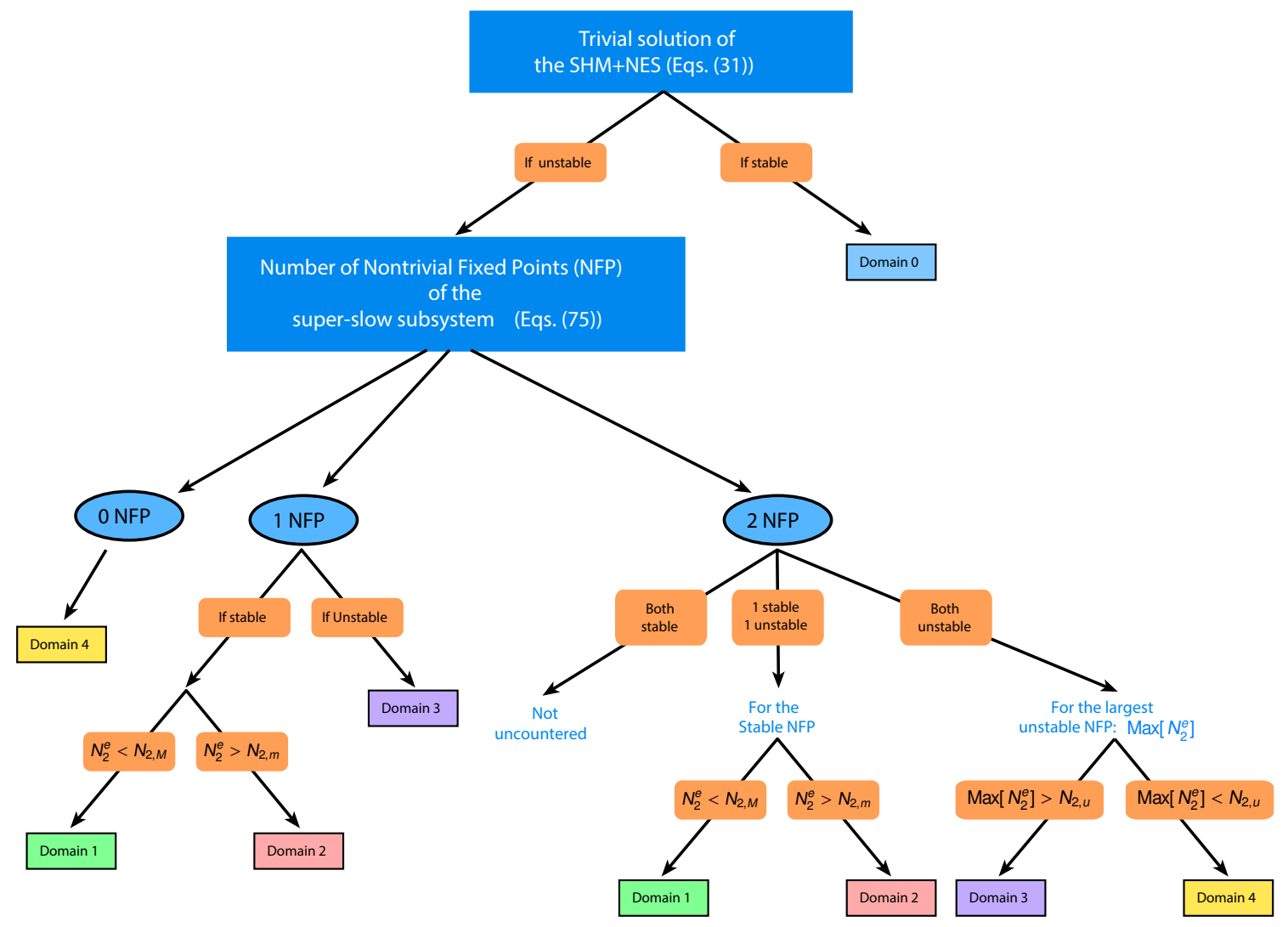

Figure 9: Algorithm for the determination of the domain of existence of the steady-state regimes of the SHM+NES (34). Each domain is described precisely in Sects. 5.1 to 5.4.

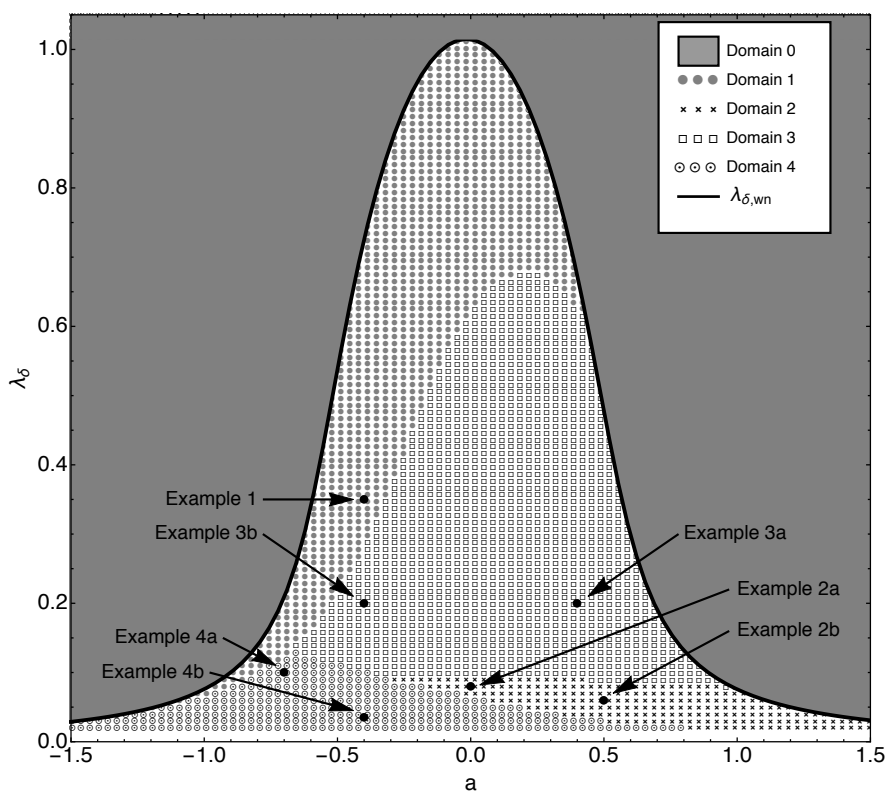

Figure 10: Prediction, in the plane $\left(\lambda_{\delta}, a\right)$, of the domains of existence of the four steady-regimes: Complete suppression (the gray area outside the curve $\lambda_{\delta, \mathrm{wn}}(a)$ ), Partial suppression through Periodic Response $(\bullet)$, Partial suppression through Strongly Modulated Response ("ם") and no suppression ("๑"). Parameters used: see Eq. (85).

see that the full slow-flow system never reach these streamlines and sustained relaxation oscillations are observed.
To know what situation will actually be observed, global stability of the SMR should be investigated. To achieve this, Starosvetsky and Gendelman [10] propose the method of $1 D$ mapping. Unfortunately, the lack of information on the amplitude of $N_{3}$ when SMR are observed makes this method unsuitable here (see the last $\S$ of Sect. 4.3.2 for a more detailed explanation).

\subsection{Domain 3: partial suppression through SMR}

This domain is represented by squares ("ه") in Fig. 10 and corresponds to two situations. In the first situation, it exists one fixed point and it is unstable. In the second situation, there are two unstable fixed points and the larger of them should satisfied the following condition: $N_{2}^{e}>N_{2, u}$. Example $3 \mathrm{a}$ and Example $3 \mathrm{~b}$ illustrate these two situations respectively (see Figs. 14 and 15). One can see in Figs. 14(b) and 15(b) that, in these situations, the fold points are reached by the system giving rise to relaxation oscillations of the slow-flow system which correspond to SMR for the $\mathrm{SHM}+\mathrm{NES}$.

\subsection{Domain 4: no suppression}

This domain is represented by dotted circles ("๑") in Fig. 10 and corresponds to two situations. The first situation corresponds to the case of two unstable fixed points and for both fixed points we have: $N_{2, M}<N_{2}^{e}<N_{2, u}$ (see Example 4a in Fig. 16). In the second situation, the slow-flow system has no fixed points. Therefore, there exists only the trivial 
solution of the SHM+NES, and it is unstable (see Example 4b in Fig. 17). In the case of no suppression, the GR instability is to strong to be suppressed by the NES attachment through PRs or SMRs and after a transient regime an explosion of the slow-flow in finally observed (a depicted in $6(d))$.

\section{Conclusion}

In the framework of NES properties exploration, we studied the steady-state response regimes of a ground resonance helicopter model including a ungrounded NES on the fuselage. An helicopter model involving blade and fuselage dynamics was reduced applying successively Coleman and binormal transformations and coupled to an ungrounded cubic NES defining the SHM+NES (Simplest Helicopter Model + Non Linear Energy Sink) model. This model reproduces the Ground Resonance (GR) instability corresponding to frequency coalescence of the fuselage mode and the regressive rotor mode. Selecting different values of the blade damping, four steady-state response regimes were highlighted: complete suppression, partial suppression through strongly modulated response, partial suppression through periodic response and no suppression of the GR instability.

The first regime corresponds to the local stability of the trivial solution of the SHM+NES. To explain all the regimes the slow flow of the system is determined using complexification-averaging approach. The presence a small dimensionless parameter related to the mass of the NES in the slow-flow system implies that it involves one "slow" complex variable and two "super-slow" complex variables. The "super-slow/slow" nature of the system allowed us to use the so-called geometric singular perturbation theory to analyze it. In particular, the Critical Manifold of the slow flow was determined. Its shape involving two folded lines and the associated stability properties provide an analytical tool to explain and predict the existence of three regimes: periodic response regimes, strongly modulated response regimes and no suppression regimes which appear when the trivial solution is unstable.

A procedure which allow to determine the domains of existence of these regimes was proposed. This procedure was used to analysis the influence of the damping of the blades and the rotor speed on the response regimes of the SHM+NES model for a set of nominal numerical values of the other parameters of the SHM+NES model. In the unstable trivial solution area, four regimes were predicted: partial suppression through periodic response, partial suppression through strongly modulated response, partial suppression through periodic response or strongly modulated response and no suppression. All these regimes were validated from direct numerical integration of the SHM+NES model.

The analytical nature of this approach makes possible other parametric studies. for example with respect to the NES parameters in order to know if it is possible to design a NES compatible with engineering applications.

\section{Acknowledgments}

The authors wish to thank Mr. François Malburet for helpful discussions and suggestions. This research is done within the framework of the industrial chair "Dynamique des Systèmes Mécaniques Complexes (Dynamics of complex mechanical systems)" financed by the Airbus Group Foundation.

\section{A About the link between the periodic so- lutions of the SHM+NES and the fixed points of the complexified-averaged sys- tem}

Let's consider a periodic steady-state regime of the system (34) defined by

$$
\begin{aligned}
v_{p}(t) & =V \sin \left(\left(\omega_{y}+\Delta \omega\right) t+\theta_{v}\right) \\
w_{p}(t) & =W \sin \left(\left(\omega_{y}+\Delta \omega\right) t+\theta_{w}\right) \\
q_{1, p}(t) & =Q_{1} \exp \left[j\left(\left(\omega_{y}+\Delta \omega\right) t+\theta_{q_{1}}\right)\right],
\end{aligned}
$$

where $V, W, Q_{1}, \theta_{y}, \theta_{h} \theta_{q_{1}}$ and $\Delta \omega$ are real constant. $\Delta \omega$ characterizes the error made using $\omega_{y}$ as fast component frequency in the complexification-averaging method. Even if this error is very small, it must be taken into account to obtain an accurate definition of the periodic regimes of Eqs. (34) from slow-flow system (39).

Through Eqs. (35), (38), (86), (87) and (88), we have

$$
\begin{aligned}
\phi_{1} & =\left(\dot{v}_{p}+j \omega_{y} v_{p}\right) e^{-j \omega_{y} t} \\
& =V\left(\omega_{y}+\frac{\Delta \omega}{2}\right) e^{j\left(\Delta \omega t+\theta_{v}\right)}+\text { t.h.f, } \\
\phi_{2} & =\left(\dot{w}_{p}+j \omega_{y} w_{p}\right) e^{-j \omega_{y} t} \\
& =W\left(\omega_{y}+\frac{\Delta \omega}{2}\right) e^{j\left(\Delta \omega t+\theta_{w}\right)}+\text { t.h.f, }
\end{aligned}
$$

where "t.h.f" means term of higher frequency, and finally,

$$
\begin{aligned}
\phi_{3} & =q_{1, p} e^{-j \omega_{y} t} \\
& =Q_{1} e^{j\left(\Delta \omega t+\theta_{q_{1}}\right)} .
\end{aligned}
$$

Looking at Eqs. (89), (90) and (91), it is easy to show that the periodic solutions of Eqs. (34) cannot be defined directly as the fixed point of the complexified-averaged system (39) (i.e. as $\dot{\phi}=0$ ). Indeed, the frequency error $\Delta \omega$ caused a linear growth of the arguments of the complex amplitude which are therefore not stationary. However, the slope $\Delta \omega$ of this linear variation is the same for each variable $\phi_{i}$. Therefore, from the polar form $\phi_{i}(t)=n_{i}(t) e^{j \theta_{i}(t)}$, the argument differences

$$
\left\{\begin{array}{l}
\delta_{21}=\theta_{2}(t)-\theta_{1}(t)=\theta_{w}-\theta_{v} \\
\delta_{31}=\theta_{3}(t)-\theta_{1}(t)=\theta_{q_{1}}-\theta_{v}
\end{array}\right.
$$

(where the component $\phi_{1}(t)$ is chosen arbitrary as a master 

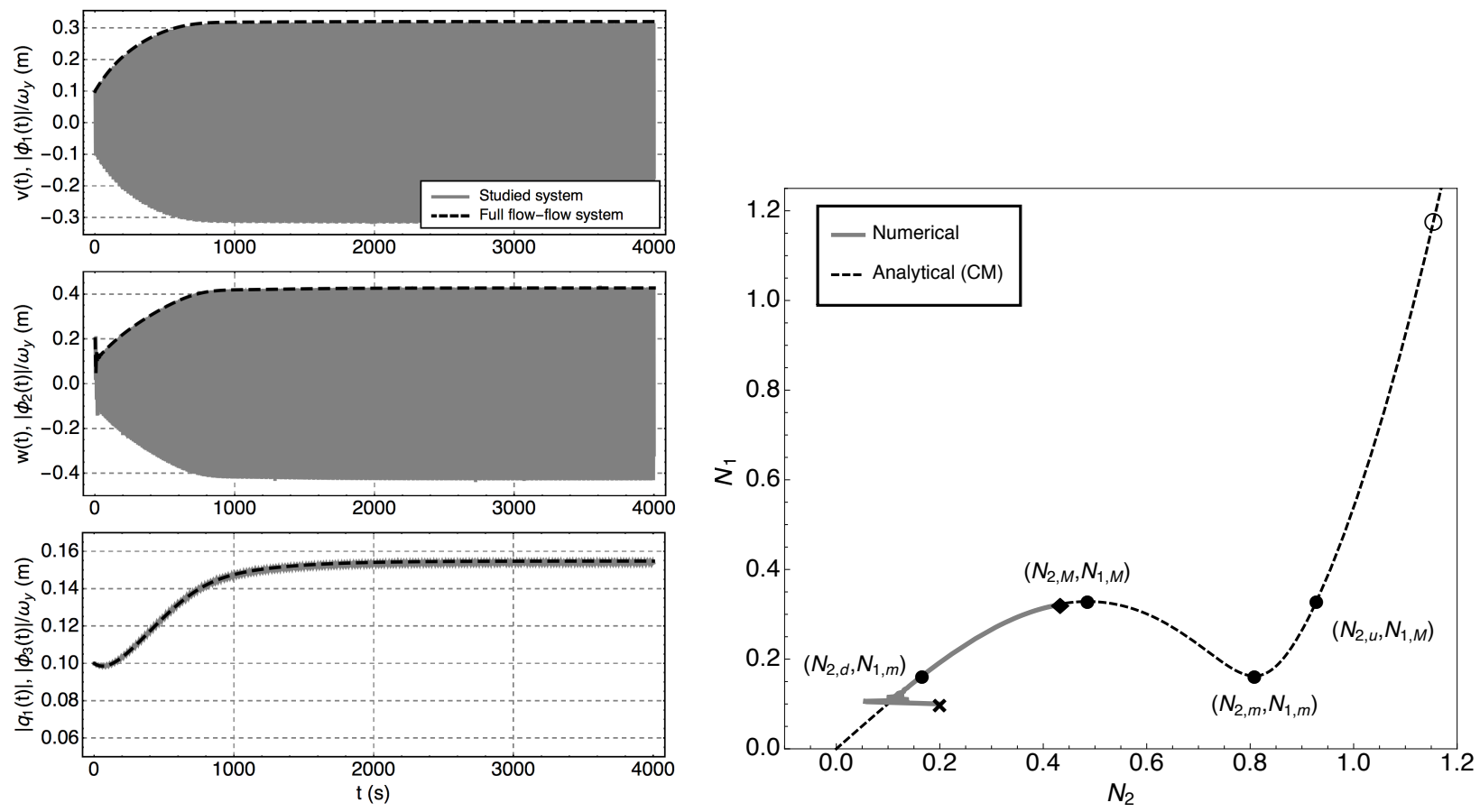

(a)

(b)

Figure 11: Example 1. Parameters used: see Eq. (85), $a=-0.4$ and $\lambda_{\delta}=0.35$. (a) Comparison between numerical simulation of the SHM+NES (34) (gray solid line)) and the full slow-flow system (49) (black dashed line). (b) Comparison between the trajectory of the simulated slow-flow system (49) in the plane $\left(N_{2}, N_{1}\right)$ and the Critical Manifold (58a). "๑": position ( $\left.N_{2, M}, N_{1, M}\right)$, $\left(N_{2, m}, N_{1, m}\right),\left(N_{2, u}, N_{1, M}\right)$ and $\left(N_{2, d}, N_{1, m}\right), \diamond$ : stable fixed points, " $\bigcirc$ ": unstable fixed points and $\times$ : initial conditions.

component) are stationary. Therefore, the periodic solutions of the system of Eqs. (34) may be defined from the complexified-averaged system (39) as the nontrivial fixed points of the system of differential equations describing the dynamic of the variables $n_{1}, n_{2}, n_{3}, \delta_{21}$ and $\delta_{31}$.

\section{References}

[1] R. P. Coleman, A. M. Feingold, Theory of self excited mechanical oscillations of helicopter rotor with hinged blades, Tech. rep., NACA Report 1351 (1958).

[2] W. Johnson, Helicopter theory, Dover publications, inc., 1994.

[3] A. R. S. Bramwell, D. Balmford, G. T. S. Done, Bramwell's helicopter dynamics, 2001.

[4] T. Krysinski, F. Malburet, Instabilité mécanique: contrôle actif et passif, Lavoisier, 2009.

[5] L. Sanches, G. Michon, D. Berlioz, A.and Alazard, Parametrically excited helicopter ground resonance dynamics with high blade asymmetries, Journal of Sound and Vibration 331 (16) (2012) 3897-3913.

[6] G. T. S. Done, A simplified approach to helicopter ground resonance, Aeronautical Journal 78 (761) (1974) 204-208.

[7] A. F. Vakatis, O. V. Gendelman, L. A. Bergman, D. M. McFarland, G. Kerschen, Y. S. Lee, Nonlinear Tar- geted Energy Transfer in Mechanical and Structural Systems, Springer-Verlag, Berlin, New York, 2008.

[8] R. Bellet, B. Cochelin, P. Herzog, P.-O. Mattei, Experimental study of targeted energy transfer from an acoustic system to a nonlinear membrane absorber, Journal of Sound and Vibration 329 (2010) 2768-2791.

[9] A. Vakakis, O. Gendelman, Energy pumping in nonlinear mechanical oscillators: Part II - Resonance capture, Journal of Applied Mechanics 68 (2001) 42-48.

[10] Y. Starosvetsky, O. V. Gendelman, Strongly modulated response in forced 2dof oscillatory system with essential mass and potential asymmetry, Physica D 237 (13) (2008) 1719-1733. doi:10.1016/j.physd. 2008.01 .019$.

[11] L. Manevitch, Complex representation of dynamics of coupled nonlinear oscillators, in: L. Uvarova, A. Arinstein, A. Latyshev (Eds.), Mathematical Models of Non-Linear Excitations, Transfer, Dynamics, and Control in Condensed Systems and Other Media, Springer US, 1999, pp. 269-300. doi:10.1007/ 978-1-4615-4799-0\_24.

[12] E. Gourc, S. Seguy, G. Michon, A. Berlioz, Chatter Control in Turning Process with a Nonlinear Energy Sink, Advanced Materials Research 698 (2013) 89-98. doi:10.4028/www.scientific.net/AMR . 698.89.

[13] S. Bab, S. E. Khadem, M. Shahgholi, Lateral vibration attenuation of a rotor under mass eccentricity force 

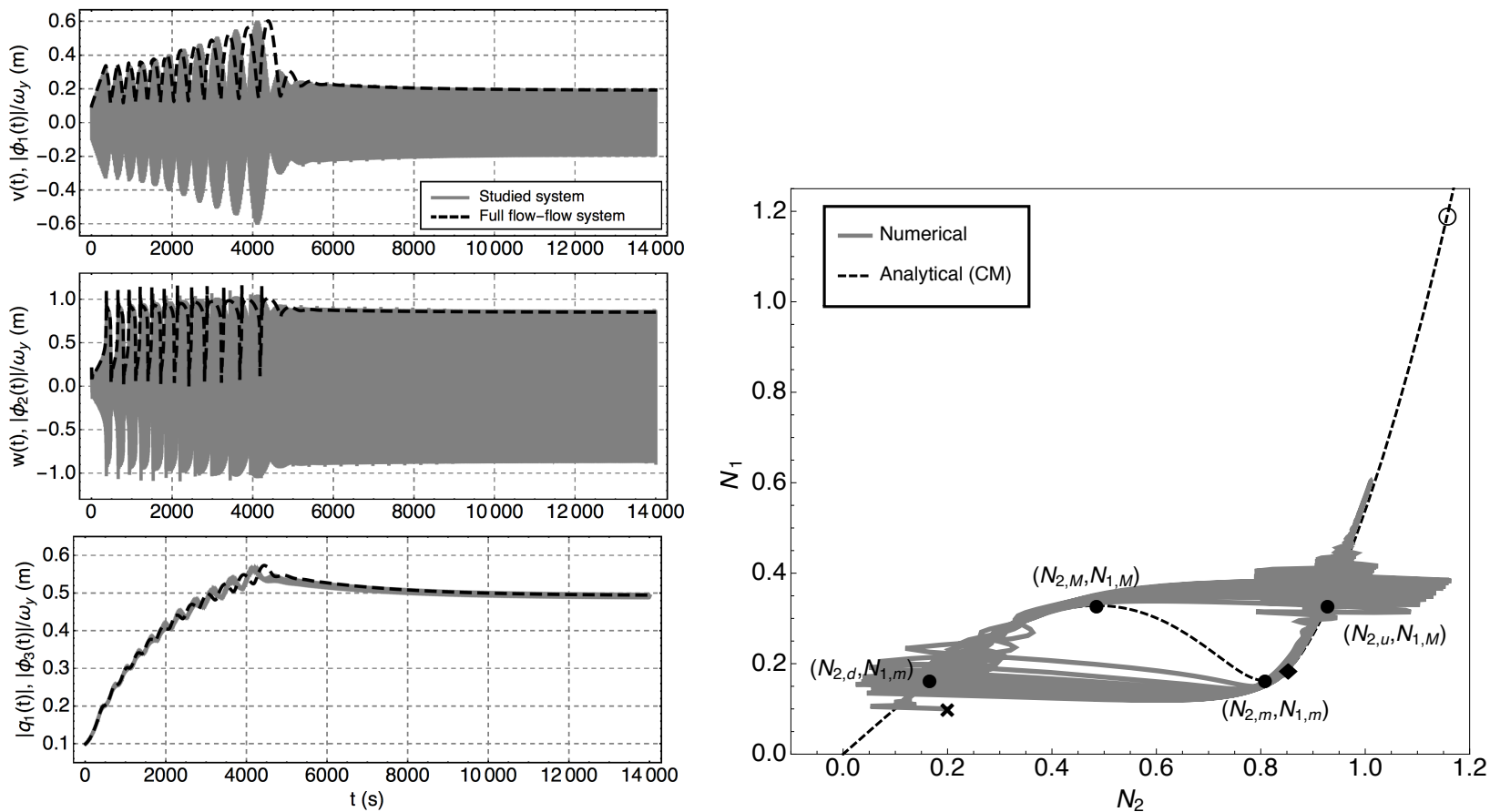

(a)

(b)

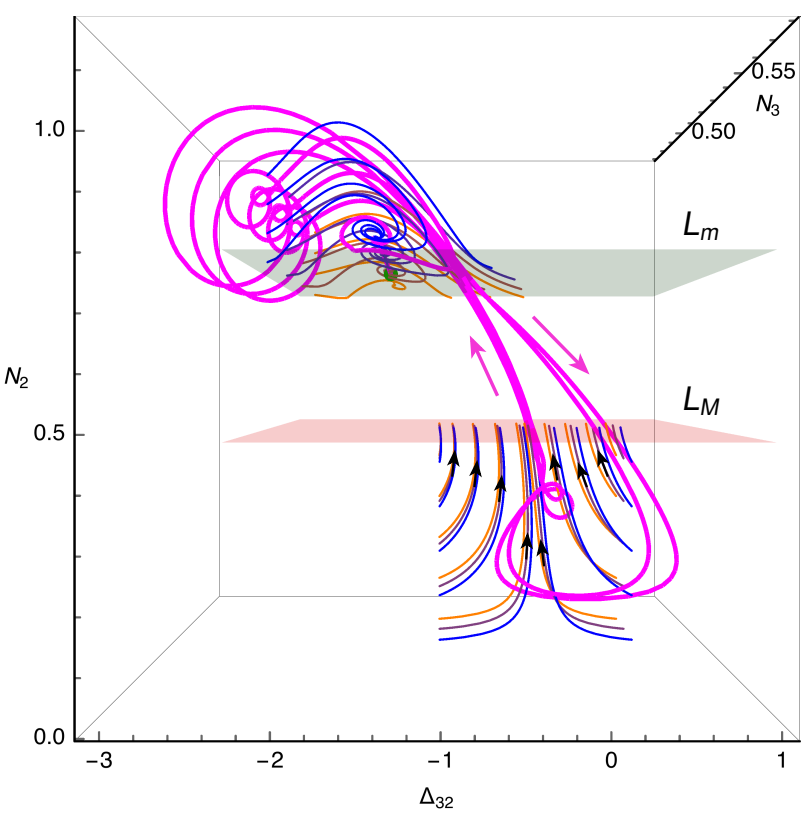

(c) Top view

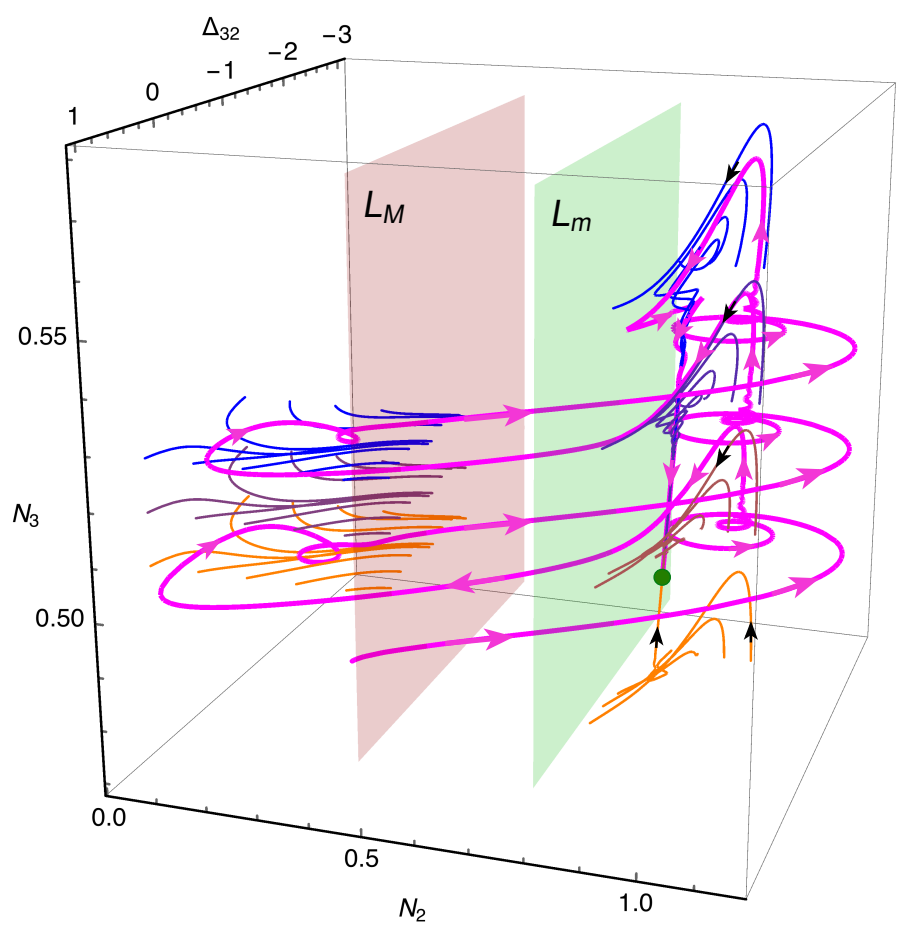

(d) Optimized view

Figure 12: Example 2a. Parameters used: see Eq. (85), $a=0$ and $\lambda_{\delta}=0.08$. (a) and (b) same caption as for Fig. 11. (c) and (d) Phase portraits of the desingularized super-slow subsystem (76) (shaded solid lines from orange to blue, only streamlines in the stable part of the CM are shown) compared to the trajectory of the full slow-flow system (49) (solid magenta lines) in the $\left(\Delta_{32}, N_{2}, N_{3}\right)$-space. Green disk marker indicates the position of the stable fixed point and arrows indicates the direction of the flow. The trajectory of full slow-flow system (49) is only plotted from $t=3250 \mathrm{~s}$ to the end $(t=14000 \mathrm{~s})$. 

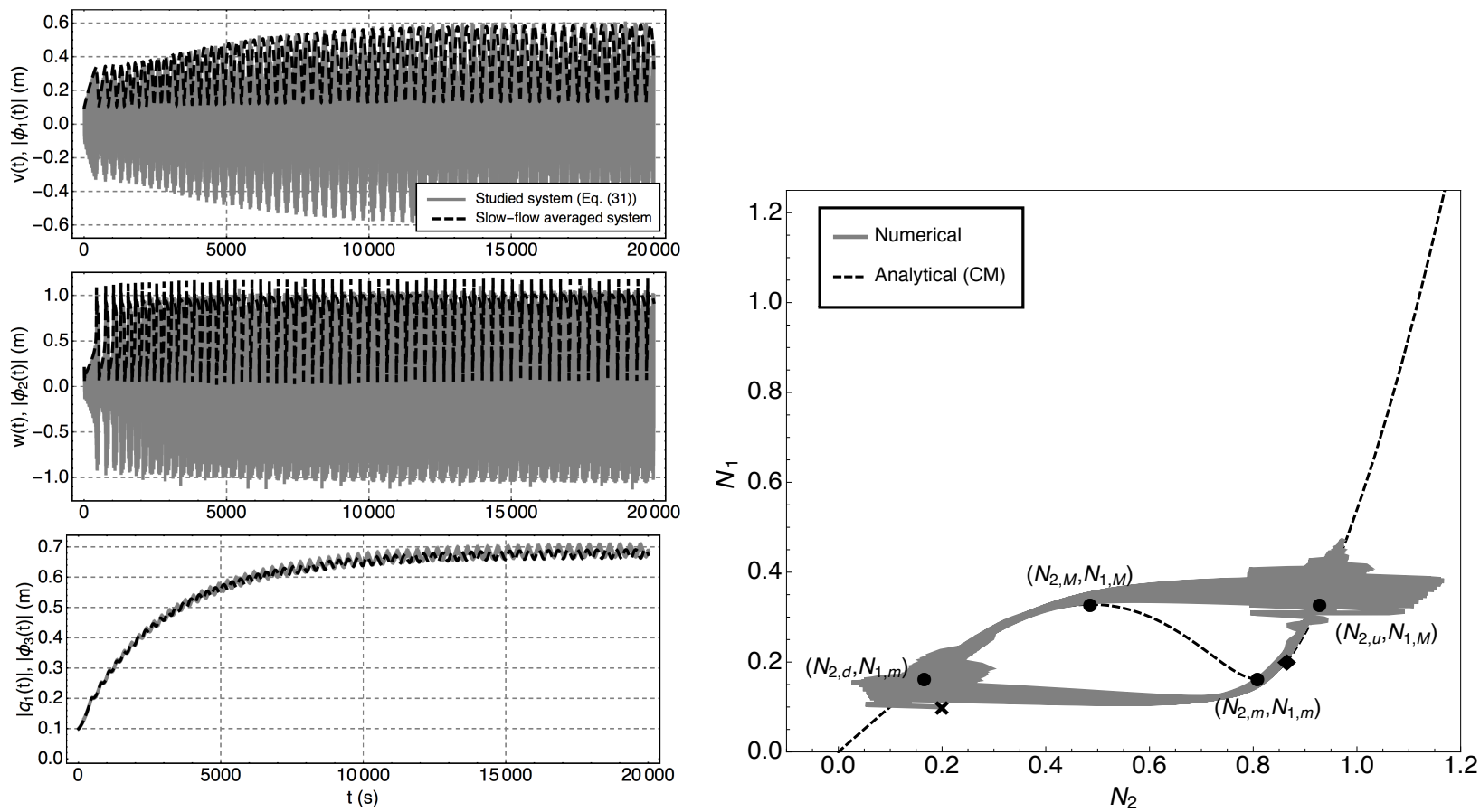

(a)

(b)

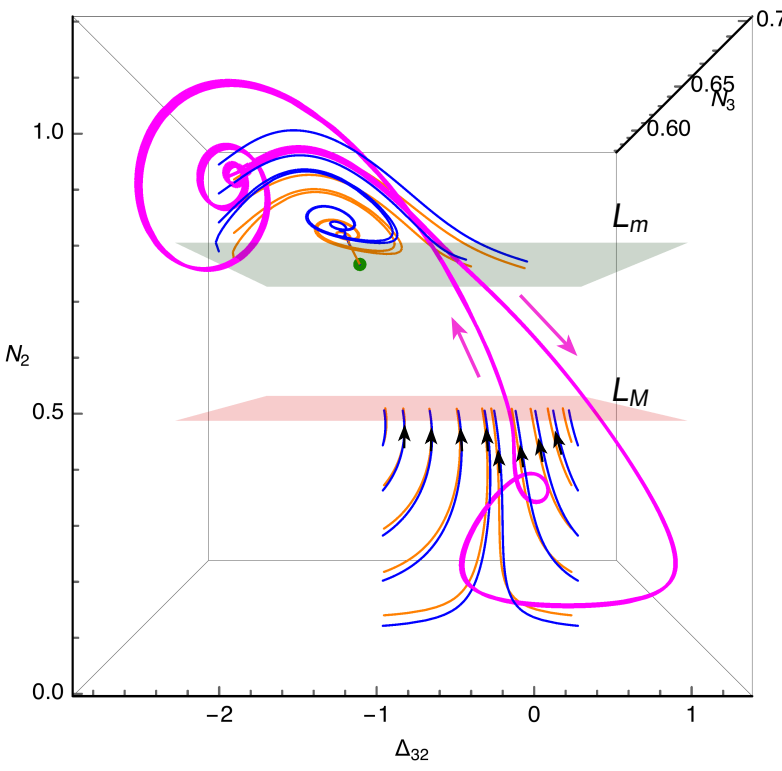

(c) Top view

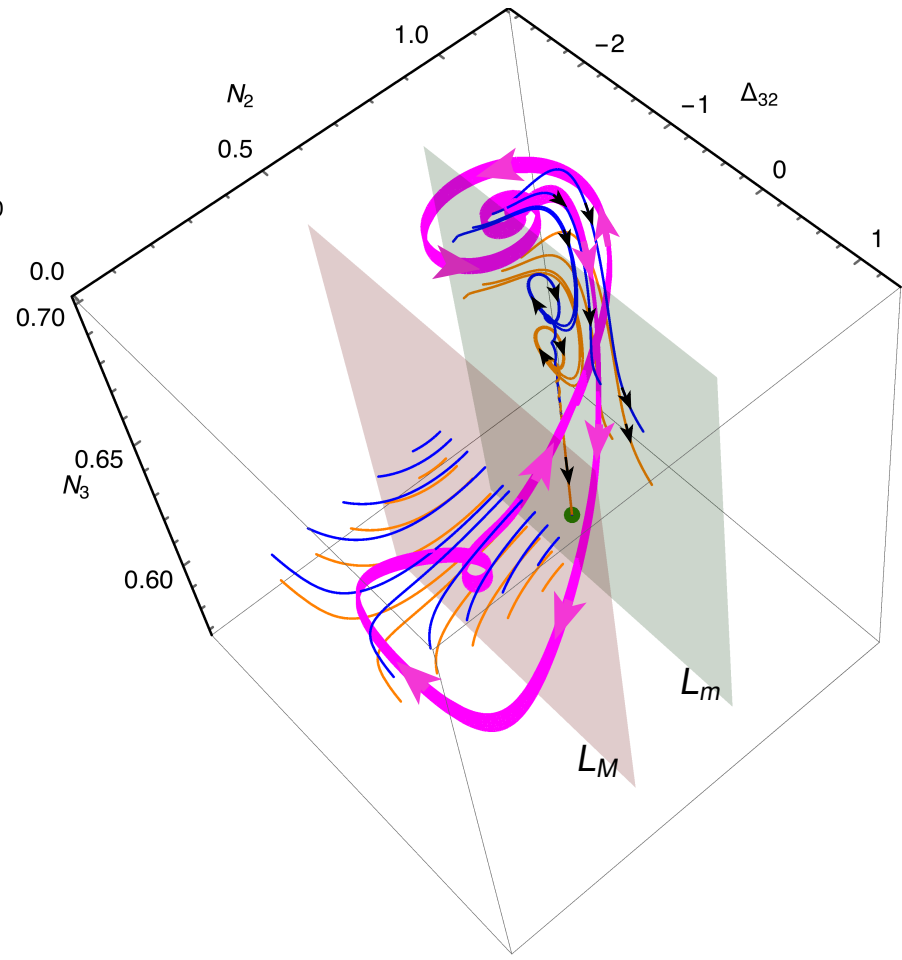

(d) Optimized view

Figure 13: Example 2b. Parameters used: see Eq. (85), $a=0.5$ and $\lambda_{\delta}=0.06$. Same caption as for Fig. 11. (c) and (d) Phase portraits of the desingularized super-slow subsystem (76) (shaded solid lines from orange to blue, only streamlines in the stable part of the CM are shown) compared to the trajectory of the full slow-flow system (49) (solid magenta lines) in the $\left(\Delta_{32}, N_{2}, N_{3}\right)$-space. Green disk marker indicates the position of the stable fixed point and arrrows indicates the direction of the flow. The trajectory of full slow-flow system (49) is only plotted from $t=13000$ s to the end $(t=20000 \mathrm{~s})$. 

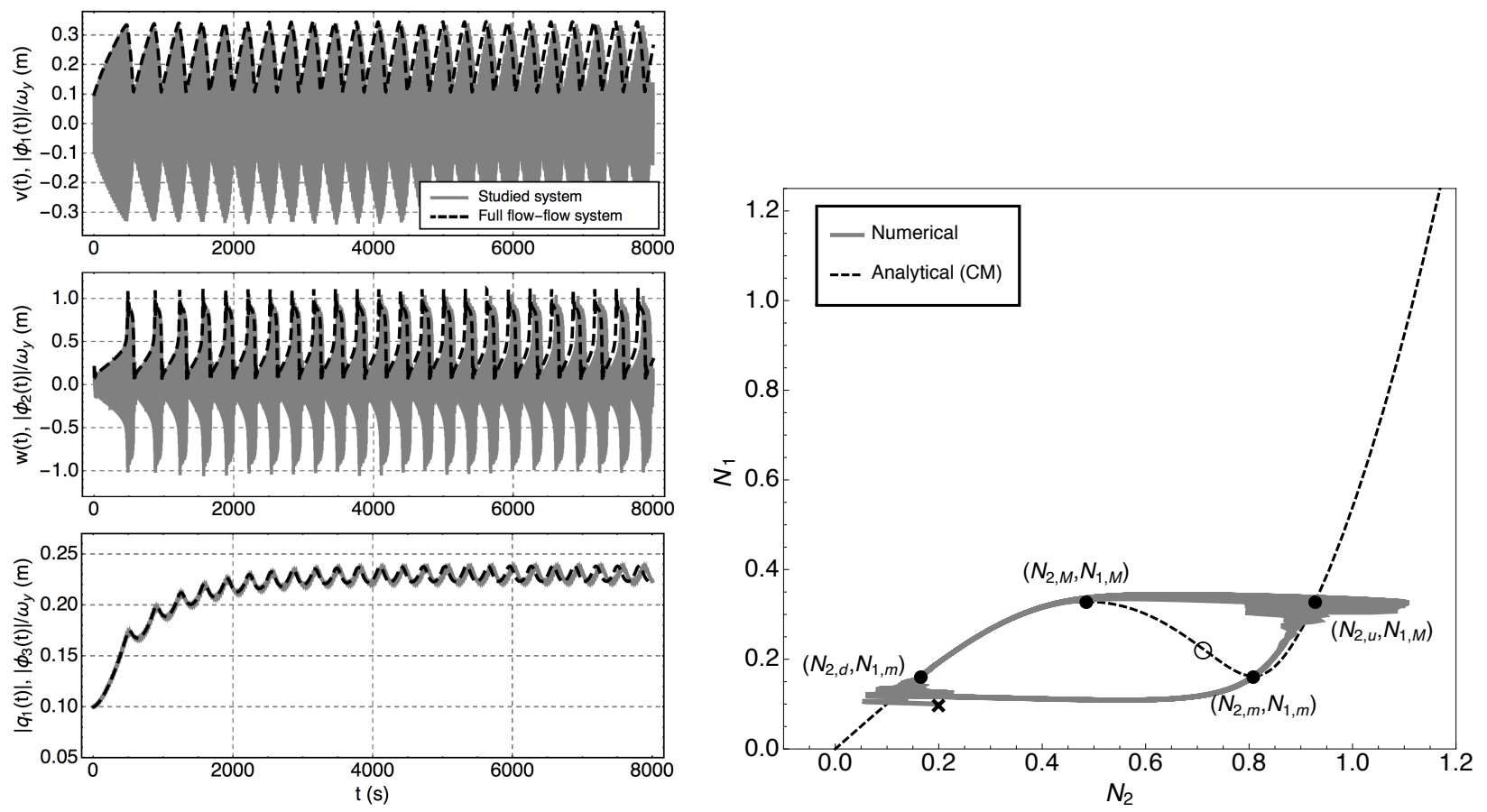

(a)

(b)

Figure 14: Example 3a. Parameters used: see Eq. (85), $a=0.4$ and $\lambda_{\delta}=0.2$. Same caption as for Fig. 11.
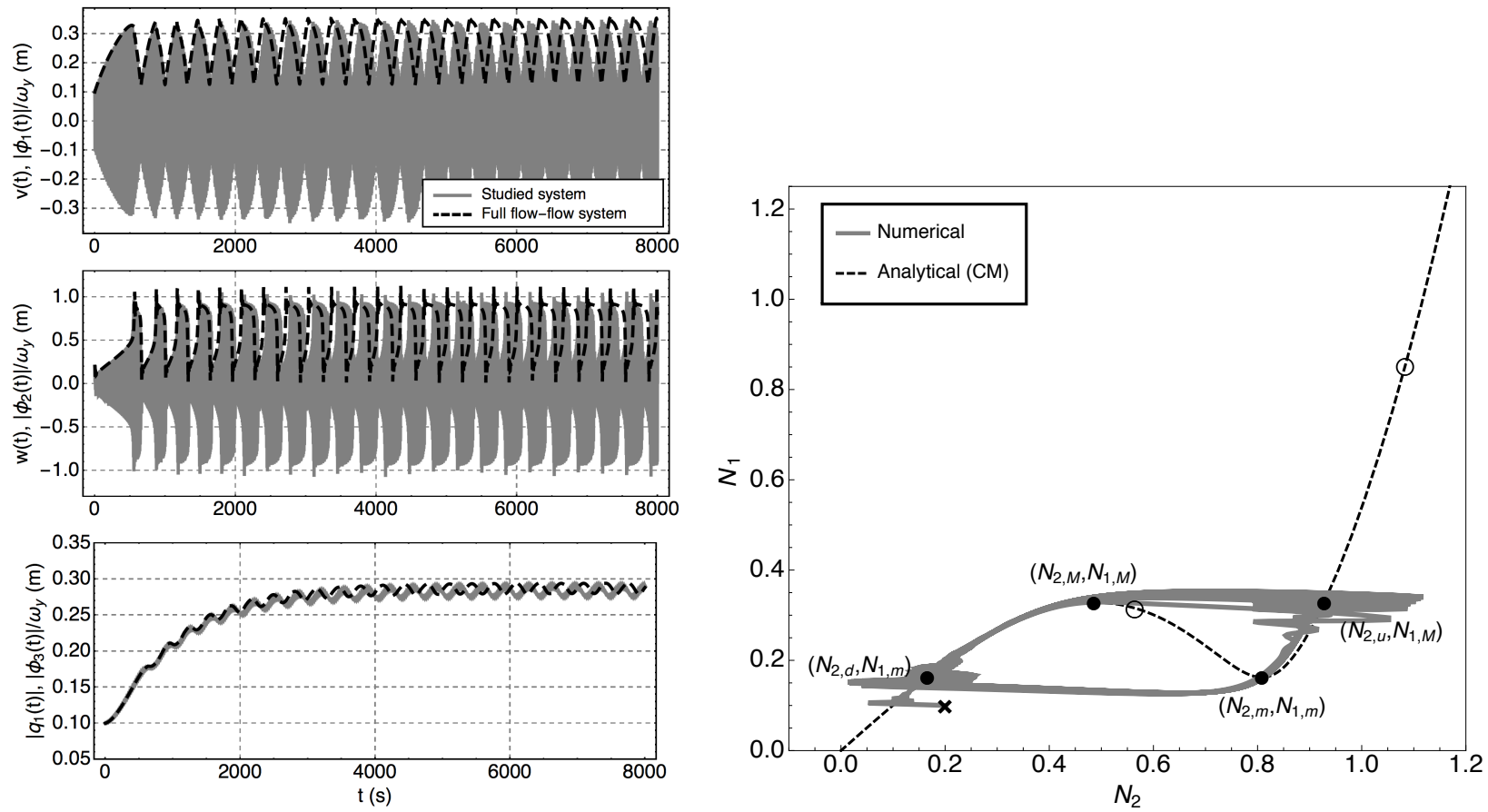

(a)

(b)

Figure 15: Example 3b. Parameters used: see Eq. (85), $a=-0.4$ and $\lambda_{\delta}=0.2$. Same caption as for Fig. 11. 

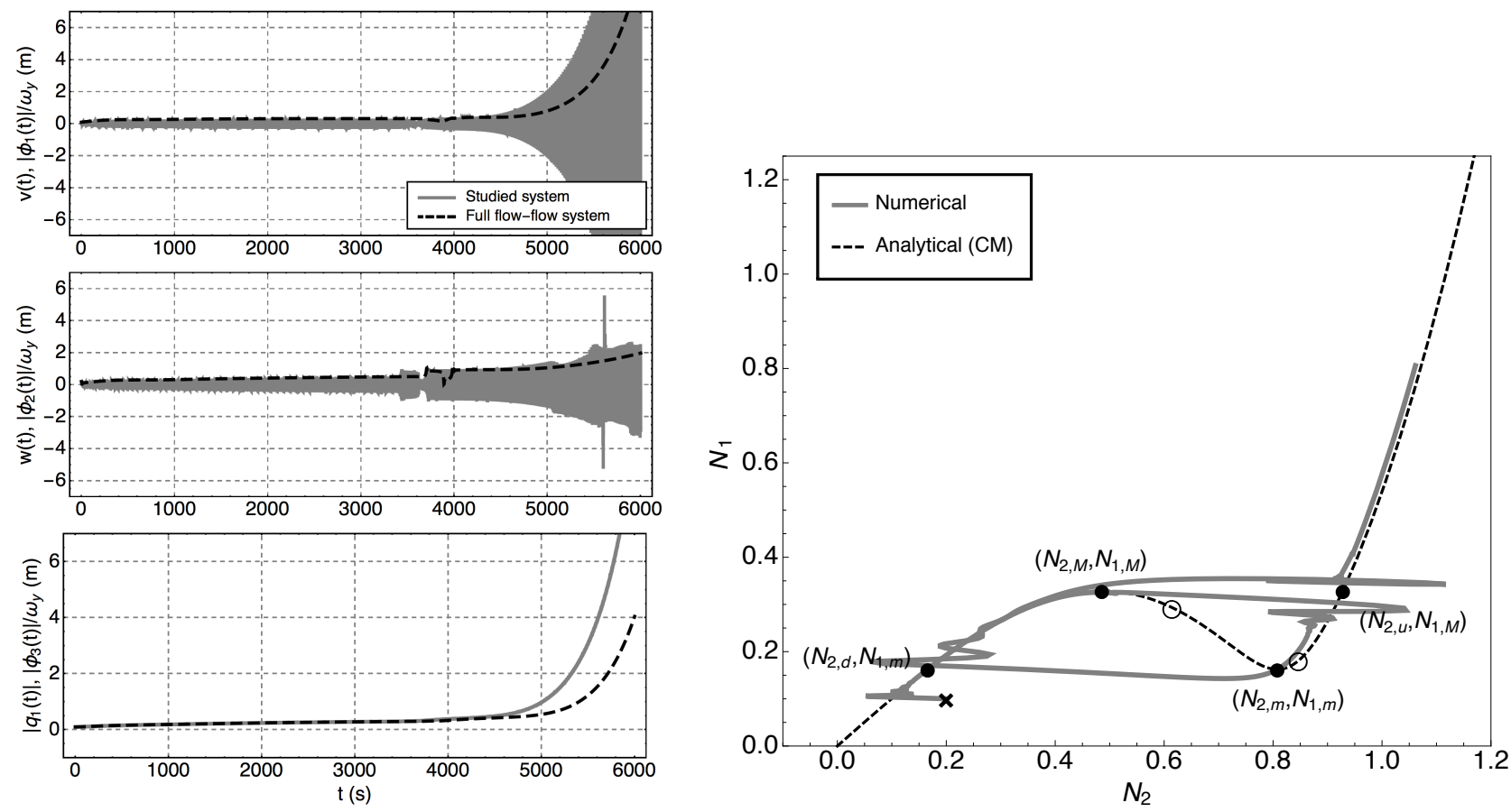

(b)

Figure 16: Example 4a. Parameters used: see Eq. (85), $a=-0.7$ and $\lambda_{\delta}=0.1$. Same caption as for Fig. 11.
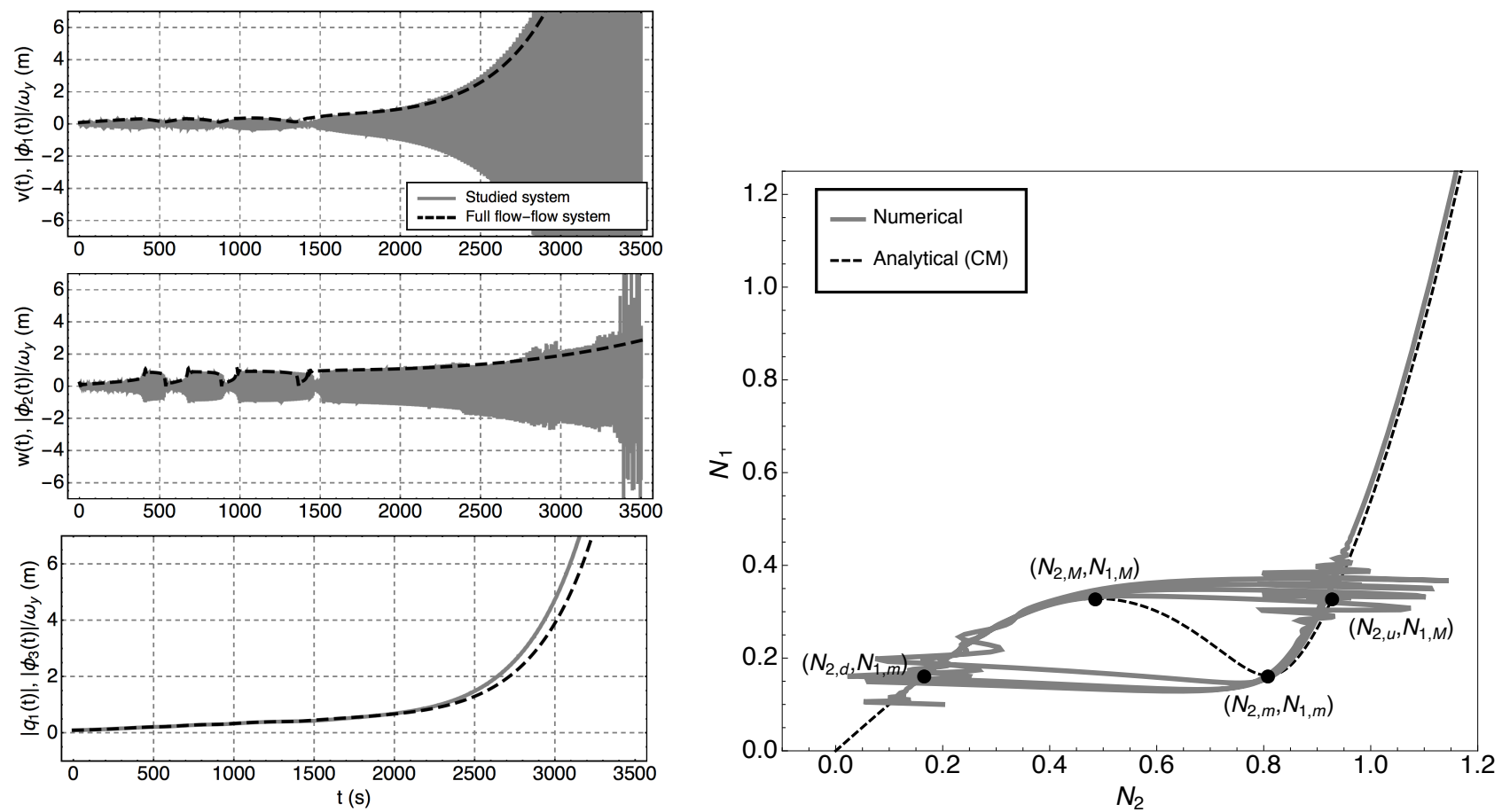

(a)

(b)

Figure 17: Example 4b. Parameters used: see Eq. (85), $a=-0.4$ and $\lambda_{\delta}=0.035$. Same caption as for Fig. 11. 
using non-linear energy sink, International Journal of Non-Linear Mechanics 67 (2014) 251-266. doi:10. 1016/j.ijnonlinmec. 2014.08.016.

[14] Y. S. Lee, A. F. Vakakis, L. A. Bergman, D. M. McFarland, Suppression of limit cycle oscillations in the van der Pol oscillator by means of passive non-linear energy sinks, Structural Control and Health Monitoring 13 (1) (2006) 41-75. doi:10.1002/stc. 143.

[15] O. V. Gendelman, T. Bar, Bifurcations of selfexcitation regimes in a Van der Pol oscillator with a nonlinear energy sink, Physica D 239 (3-4) (2010) 220229. doi:10.1016/j.physd.2009.10.020.

[16] E. Domany, O. Gendelman, Dynamic responses and mitigation of limit cycle oscillations in Van der PolDuffing oscillator with nonlinear energy sink, Journal of Sound and Vibration 332 (21) (2013) 5489-5507. doi : $10.1016 / j \cdot j s v .2013 .05 .001$.

URL http://linkinghub.elsevier.com/retrieve/ pii/S0022460X13004069

[17] Y. S. Lee, A. F. Vakakis, L. A. Bergman, D. M. McFarland, G. Kerschen, Suppression aeroelastic instability using broadband passive targeted energy transfers, part 1: Theory, AIAA Journal 45 (3) (2007) 693-711. doi: $10.2514 / 1.24062$.

[18] Y. S. Lee, A. F. Vakakis, L. A. Bergman, D. M. McFarland, G. Kerschen, Suppression aeroelastic instability using broadband passive targeted energy transfers, part 2: Experiments, AIAA Journal 45 (3) (2007) 2391-2400. doi:10.2514/1.24062.

[19] O. Gendelman, A. Vakakis, L. Bergman, D. McFarland, Asymptotic analysis of passive nonlinear suppression of aeroelastic instabilities of a rigid wing in subsonic flow, SIAM Journal on Applied Mathematics 70 (5) (2010) 1655-1677. doi:10.1137/090754819.

[20] A. Luongo, D. Zulli, Aeroelastic instability analysis of nes-controlled systems via a mixed multiple scale/harmonic balance method, Journal of Vibration and Control 20 (13) (2014) 1985-1998.

[21] O. V. Gendelman, Targeted energy transfer in systems with external and self-excitation, Proceedings of the Institution of Mechanical Engineers, Part C: Journal of Mechanical Engineering Science 225 (9) (2011) 2007-2043. doi : 10.1177/0954406211413976.

URL http://pic.sagepub.com/content/225/9/ 2007. abstract

[22] N. Fenichel, Geometric singular perturbation theory for ordinary differential equations, Journal of Differential Equations 98 (1979) 53-98.

[23] A. W. Leissa, On a curve veering aberration (ZAMP), Journal of Mathematics and Physics 25 (1974) 99-111.

[24] J. R. Kuttler, V. G. Sigillito, On curve veering, Journal of Sound and Vibration 75 (4) (1981) 585-588. doi: 10.1016/0022-460X (81) 90448-X.
[25] R. L. Halfman, Dynamics Vol. II: Systems, Variational Methods, and Relativity, Addison-Wesley Publishing Co., Inc., Cambridge, 1962.

[26] T. K. Caughey, M. E. J. O'Kelly, General theory of vibration of damped linear dynamic systems, Dynamics Laboratory, California Institute of Technology, Pasadena, California, 1963.

URL http://authors.library.caltech.edu/ 26463/

[27] C. Jones, Geometric singular perturbation theory, in: R. Johnson (Ed.), Dynamical Systems, Vol. 1609 of Lecture Notes in Mathematics, Springer Berlin Heidelberg, 1995, pp. 44-118. doi:10.1007/BFb0095239.

[28] M. Desroches, J. Guckenheimer, B. Krauskopf, C. Kuehn, H. Osinga, M. Wechselberger, Mixed-mode oscillations with multiple time scales, SIAM Review 52 (2) (2012) 211-288. doi:10.1137/100791233.

[29] R. Seydel, Practical Bifurcation and Stability Analysis, 3rd Edition, Vol. 5 of Interdisciplinary Applied Mathematics, Springer, 2010.

[30] J. Grasman, Asymptotic Methods for Relaxation Oscillations and Applications, Vol. 63 of Applied Mathematical Sciences, Springer-Verlag, 1987. 